\title{
SEMANTICS OF HIGHER-ORDER RECURSION SCHEMES
}

\author{
JIŘÍ ADÁMEK ${ }^{a}$, STEFAN MILIUS $^{b}$, AND JIŘÍ VELEBIL $^{c}$ \\ ${ }^{a, b}$ Institut für Theoretische Informatik, Technische Universität Braunschweig, Germany \\ e-mail address: adamek@iti.cs.tu-bs.de, mail@stefan-milius.eu \\ ${ }^{c}$ Faculty of Electrical Engineering, Czech Technical University of Prague, Prague, Czech Republic \\ e-mail address: velebil@math.feld.cvut.cz
}

\begin{abstract}
Higher-order recursion schemes are recursive equations defining new operations from given ones called "terminals". Every such recursion scheme is proved to have a least interpreted semantics in every Scott's model of $\lambda$-calculus in which the terminals are interpreted as continuous operations. For the uninterpreted semantics based on infinite $\lambda$-terms we follow the idea of Fiore, Plotkin and Turi and work in the category of sets in context, which are presheaves on the category of finite sets. Fiore et al showed how to capture the type of variable binding in $\lambda$-calculus by an endofunctor $H_{\lambda}$ and they explained simultaneous substitution of $\lambda$-terms by proving that the presheaf of $\lambda$-terms is an initial $H_{\lambda}$-monoid. Here we work with the presheaf of rational infinite $\lambda$-terms and prove that this is an initial iterative $H_{\lambda}$-monoid. We conclude that every guarded higher-order recursion scheme has a unique uninterpreted solution in this monoid.
\end{abstract}

\section{INTRODUCTION}

The present paper is a contribution to the study of the semantics of recursive definitions using category-theoretic tools and methods. Our goal is to present a category-theoretic semantics of higher-order recursion schemes in the sense of W. Damm [8]. To reach this goal we apply the theory of rational monads on a category $\mathscr{K}$, developed in our previous work [2] in order to formalize iteration in algebra, to the category

$$
\mathscr{K}=\operatorname{Set}^{\mathscr{F}} \quad(\mathscr{F}=\text { finite sets and functions })
$$

of sets in context. We use the approach to $\lambda$-calculus based on $H$-monoids in the category of sets in context due to M. Fiore, G. Plotkin and D. Turi [10]. Our main result is a description of the initial iterative $H$-monoid as the monoid of rational $\lambda$-terms, and the fact that in this monoid every higher-order recursion scheme has a unique uninterpreted solution.

We now explain the motivation of our paper in more detail. In the higher-order semantics we assume a given collection $\Sigma$ of existing programs of given types (that is, a manysorted signature of "terminals"). One recursively defines new typed programs $p_{1}, \ldots, p_{n}$

\footnotetext{
Key words and phrases: Higher-order recursion schemes, infinite $\lambda$-terms, sets in context, rational tree.
}

${ }^{c}$ Supported by the grant MSM 6840770014 of the Ministry of Education of the Czech Republic.

DOI:10.2168/LMCS-7 (1:15) 2011

C. J. Adámek, S. Milius, and J. Velebil

(a) Creative Commons 
(forming a many-sorted signature of "nonterminals") using symbols from $\Sigma$ and $\left\{p_{1}, \ldots, p_{n}\right\}$. If the recursion only concerns application, we can formalize this as a collection of equations

$$
p_{i}=f_{i} \quad(i=1, \ldots, n)
$$

whose right-hand sides $f_{i}$ are terms in the signature of all terminals and all non-terminals. Such collections are called (first-order) recursion schemes and were studied in 1970's by various authors, e.g. B. Courcelle, M. Nivat and I. Guessarian (see the monograph [12] and references there) or S. J. Garland and D. C. Luckham [11]. Recently, a categorical approach to semantics of first-order recursion schemes was presented by S. Milius and L. Moss [18]. In the present paper we take a first step in an analogous approach to the semantics of higher-order recursion schemes in which $\lambda$-abstraction is also used as one of the operations. That is, a higher-order recursion scheme, as introduced by W. Damm [8] (see also the recent contributions [7] and [19]) is a collection of equations $p_{i}=f_{i}$ where $f_{i}$ are terms using application and $\lambda$-abstraction on symbols from $\Sigma$ and $\left\{p_{1}, \ldots, p_{n}\right\}$. As in [18], we first study the uninterpreted semantics, where the given system is regarded as a purely syntactic construct. At this stage the operation symbols in $\Sigma$ as well as $\lambda$-abstraction and application have no interpretation on actual data. So the semantics is provided by formal (infinite) terms. These terms can be represented by rational trees, i. e., infinite trees having finitely many subtrees. Thus the uninterpreted solution assigns to each of the recursive variables $p_{i}$ in (1.1) a rational tree $p_{i}^{\dagger}$ such that the formal equations become identities if we substitute $p_{i}^{\dagger}$ for $p_{i}(i=1, \ldots, n)$. We assume $\alpha$-conversion (renaming of bound variables) but no other rules in the uninterpreted semantics. We next turn to an interpreted semantics. Here a recursion scheme is given together with an interpretation of all symbols from $\Sigma$ as well as $\lambda$-abstraction and application. Following D. Scott, we interpret the $\lambda$-calculus on a CPO, say $D$. The symbols of $\Sigma$ are interpreted as continuous operations on $D$, and formal $\lambda$-abstraction and application are the actual $\lambda$-abstraction and application in the model $D$. An interpreted solution in $D$ then assigns to each $p_{i}$ in the context $\Gamma$ of all free variables in (1.1) an element of $\mathbf{C P O}\left(D^{\Gamma}, D\right)$ (continuously giving to each assignment of free variables in $D^{\Gamma}$ an element of $D$ ) such that the formal equations in the recursion scheme become identities in $D$ when the right-hand sides are interpreted in $D$.

Example 1.1. The fixed-point operator $Y$ is specified by

$$
Y=\lambda f . f(Y f)
$$

and the uninterpreted semantics is the rational tree

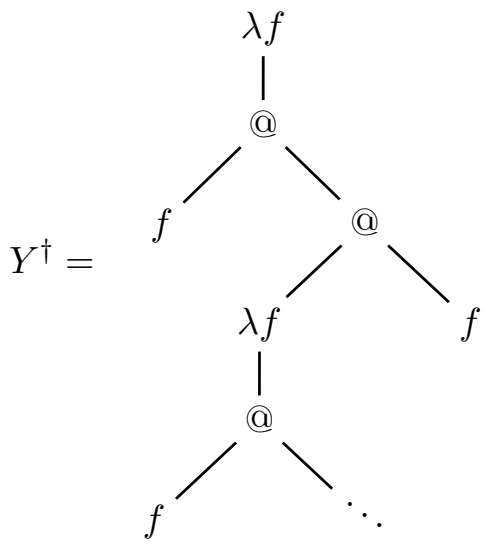


(The symbol @ makes application explicit.) The interpreted solution in $D$ is the least fixed point operator (considered as an element of $D$ ).

The above example is untyped, and indeed we are only treating the untyped case in the present paper since its uninterpreted semantics is technically simpler than the typed case; however, the basic ideas of uninterpreted semantics are similar. In contrast, the interpreted semantics (based on a specified model of $\lambda$-calculus with "terminal" symbols interpreted as operations) is more subtle in the untyped case.

Our main result is that every guarded higher-order recursion scheme has a unique uninterpreted solution, and a least interpreted one. This demonstrates that the methods for iteration in locally finitely presentable categories developed in [2] can serve not only for first-order iteration, when applied to endofunctors of Set, but also for higher-order iteration: it is possible to apply these methods to other categories, here the category of sets in context.

Related Work. This is an extended and revised version of the conference paper [4]. In addition to the material in that extended abstract we include here the theory of iterative monoids in a monoidal category, see Section 4 below, and we provide detailed proofs.

\section{Presheaves as Algebras}

Notation 2.1.

(1) Throughout the paper a given countably infinite set Var of variables is assumed. Finite subsets $\Gamma \subseteq$ Var are called contexts and form a full subcategory $\mathscr{F}$ of Set. We also assume that a (possibly empty) finitary signature $\Sigma$ is given.

When speaking about formulas in context $\Gamma$ we mean those that have all free variables in $\Gamma$. For example, $\lambda x . y x$ is a formula in context $\Gamma=\left\{y, y^{\prime}\right\}$.

(2) The category Set ${ }^{\mathscr{F}}$ of "covariant presheaves" on $\mathscr{F}$ is well known to be equivalent to the category of finitary endofunctors of Set. Indeed, every endofunctor $X$ yields the presheaf $X \uparrow \mathscr{F}$, and conversely, every presheaf $X$ in Set $^{\mathscr{F}}$ has a left Kan extension to a finitary endofunctor of Set: for every set $M$ we have

$$
X(M)=\bigcup X i_{\Gamma}[X(\Gamma)]
$$

where the union ranges over embeddings $i_{\Gamma}: \Gamma \hookrightarrow M$ of contexts $\Gamma$ into $M$, and $X i_{\Gamma}[X(\Gamma)]$ denotes the image of $X i_{\Gamma}$.

(3) From now on we speak about presheaves when objects of Set $^{\mathscr{F}}$ are meant. The word endofunctor is reserved for endofunctors on $\mathbf{S e t}{ }^{\mathscr{F}}$ throughout our paper.

\section{Example 2.2.}

(i) The presheaf of variables, $V$, is our name for the embedding $\mathscr{F} \hookrightarrow$ Set: $V(\Gamma)=\Gamma$. As we will see in Section 3, $V$ is the unit of the monoidal operation of substitution.

(ii) Free presheaf on one generator of context $\Gamma$ is our name for the representable presheaf

$$
\mathscr{F}(\Gamma,-) \text {. }
$$

Indeed, the Yoneda lemma states that this presheaf is freely generated by the element $\operatorname{id}_{\Gamma}$ of context $\Gamma$ : for every presheaf $X$ and every $x \in X(\Gamma)$ there exists a unique morphism $f: \mathscr{F}(\Gamma,-) \rightarrow X$ with $f_{\Gamma}\left(\operatorname{id}_{\Gamma}\right)=x$. Observe that $\mathscr{F}(\Gamma,-)$ is naturally 
isomorphic to the functor $X \mapsto X^{n}$, where $n=\operatorname{card} \Gamma$ is the power of $\Gamma$. Consequently a free presheaf on $k$ generators in contexts $\Gamma_{1}, \ldots, \Gamma_{k}$ has the form

$$
\Gamma \mapsto \Gamma^{n_{1}}+\cdots+\Gamma^{n_{k}}, \quad \text { where } n_{i}=\operatorname{card} \Gamma_{i} .
$$

This is the "polynomial presheaf" $X_{\Sigma}$ of a signature $\Sigma$ of $k$ operation symbols of the given arities $n_{i}$.

(iii) The presheaf $F_{\lambda}$ of (finite) $\lambda$-terms is defined via a quotient since we want to treat $\lambda$-terms always modulo $\alpha$-conversion. We first consider the set of all $\lambda$-trees $\tau$ given by the grammar

$$
\tau::=x|\tau @ \tau| \lambda y \cdot \tau \quad(x, y \in \operatorname{Var}) .
$$

In the graphic form:

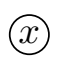

or

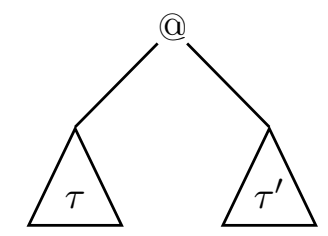

or

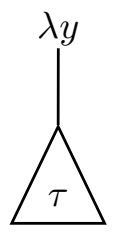

The notions of a free and bound variable of a $\lambda$-tree $\tau$ are defined as usual.

As explained in [10, the following approach is equivalent to defining $\lambda$-terms up to $\alpha$-equivalence by de Bruijn levels: We first denote by $F_{\lambda}^{\prime}(\Gamma)$ the set of all finite $\lambda$-trees with free variables in the context $\Gamma=\left\{x_{1}, \ldots, x_{n}\right\}$. We then define the presheaf $F_{\lambda}$ in context $\Gamma$ by

$$
F_{\lambda}(\Gamma)=F_{\lambda}^{\prime}(\Gamma) / \sim_{\alpha}
$$

where $\sim_{\alpha}$ represents the $\alpha$-conversion: this is the least congruence with $\lambda y . \tau \sim_{\alpha}$ $\lambda z . \tau[z / y]$, where $z$ is not a free variable of $\tau$. And we define $F_{\lambda}$ on morphisms $\gamma: \Gamma \rightarrow \Gamma^{\prime}$ by choosing a term $t \in F_{\lambda}(\Gamma)$, relabelling all bound variables so that they do not lie in $\Gamma^{\prime}$, and denoting by $F_{\lambda} \gamma(t)$ the term obtained by relabelling every free variable $x \in \Gamma$ to $\gamma(x) \in \Gamma^{\prime}$.

We call the congruence classes of finite $\lambda$-trees modulo $\alpha$-conversion finite $\lambda$-terms. (Finite $\lambda$-trees do not form a presheaf, due to possible clashes of bound and free variables. For example consider the $\lambda$-tree

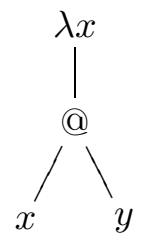

in $F_{\lambda}^{\prime}\{y\}$ and the function $j:\{y\} \rightarrow \Gamma$ with $x \in \Gamma$ and $j(y)=x$. Then to define the action of $F_{\lambda}^{\prime}$ on $j$ we must rename the bound variable $x$ to some $z \notin \Gamma$. But in fact, any other renaming to $z^{\prime} \notin \Gamma$ is fine, too. So trying to define the action of $F_{\lambda}^{\prime}$ on functions naturally forces us to consider equivalence classes modulo $\alpha$-conversion.)

(iv) The presheaf $F_{\lambda, \Sigma}$ of finite $\lambda$ - $\Sigma$-terms is defined analogously: in (2.1) we just add the term $\sigma\left(\tau_{1}, \ldots, \tau_{n}\right)$ for every $n$-ary operation symbol $\sigma \in \Sigma$, and in (2.2) the corresponding tree. 
(v) The presheaf $T_{\lambda}$ of all (finite and infinite) $\lambda$-terms is defined analogously to $F_{\lambda}$. We first denote by $T_{\lambda}^{\prime}(\Gamma)$ the set of all trees (2.2) dropping the assumption of finiteness. Then we use $\alpha$-conversion: for infinite trees $t$ and $t^{\prime}$ we write

$$
t \sim_{\alpha} t^{\prime}
$$

if their (finite) cuttings at level $k$ (with label $\perp$ for all leaves at level $k$ ) are $\alpha$-equivalent in the above sense for all $k \in \mathbb{N}$. (We can formalize this by using $\Sigma_{\perp}=\Sigma \cup\{\perp\}$ with $\perp$ a constant symbol outside of $\Sigma \cup \operatorname{Var}$ ). The presheaf $T_{\lambda}$ is defined on objects $\Gamma$ by $T_{\lambda}(\Gamma)=T_{\lambda}^{\prime}(\Gamma) / \sim_{\alpha}$ and on morphisms $\gamma: \Gamma \rightarrow \Gamma^{\prime}$ by relabellings of variables as in (iii). Observe that since $\operatorname{Var} \backslash \Gamma$ is infinite, the relabelling of bound variables needed here causes no problem.

(vi) The presheaf $R_{\lambda}$ of rational $\lambda$-terms is also defined analogously. Recall that a tree is called rational if it has up to isomorphism only finitely many subtrees. We denote by $R_{\lambda}^{\prime}(\Gamma)$ the set of all rational trees in $T_{\lambda}^{\prime}(\Gamma)$ and define a presheaf $R_{\lambda}$ by $R_{\lambda}(\Gamma)=$ $R_{\lambda}^{\prime}(\Gamma) / \sim_{\alpha}$ on objects, and by relabellings of variables (as in (iii)) on morphisms. Observe that, by definition, every rational $\lambda$-term $t$ is represented by a rational $\lambda$ tree. However, $t$ can also be represented by non-rational $\lambda$-trees-for example, if it contains infinitely many $\lambda$ 's, the $\alpha$-conversion can introduce an infinite number of bound variables.

(vii) The presheaves $T_{\lambda, \Sigma}$ (of all $\lambda$ - $\Sigma$-terms) and $R_{\lambda, \Sigma}$ (of rational $\lambda$ - $\Sigma$-terms) are obvious modifications of (iv) and (v): one adds to (2.1) and (2.2) the case $\sigma\left(\tau_{1}, \ldots, \tau_{n}\right)$ for all $n$-ary symbols $\sigma \in \Sigma$ and all (rational) $\lambda$ - $\Sigma$-trees $\tau_{1}, \ldots, \tau_{n}$.

Notation 2.3. We denote by $\delta$ : $\operatorname{Set}^{\mathscr{F}} \rightarrow$ Set $^{\mathscr{F}}$ the endofunctor defined by

$$
\delta X(\Gamma)=X(\Gamma+1) .
$$

Observe that $\delta$ preserves limits and colimits.

Note that an algebra for $\delta$ is a presheaf $Y$ together with an operation $Y(\Gamma+1) \rightarrow$ $Y(\Gamma)$ for all contexts $\Gamma$ - this is precisely the form of $\lambda$-abstraction, where to a formula $f$ in $Y(\Gamma+\{y\})$ we assign $\lambda y . f$ in $Y(\Gamma)$. The other $\lambda$-operation, application, is simply a presheaf morphism $X \times X \rightarrow X$, that is, a binary operation on $X$. We put these two together:

Notation 2.4. Let $H_{\lambda}$ denote the endofunctor of $\mathbf{S e t}^{\mathscr{F}}$ given by

$$
H_{\lambda} X=X \times X+\delta X .
$$

Thus, an algebra for $H_{\lambda}$ is a presheaf $X$ together with operations of application $X(\Gamma) \times$ $X(\Gamma) \rightarrow X(\Gamma)$ and abstraction $X(\Gamma+1) \rightarrow X(\Gamma)$ for all contexts $\Gamma$; these operations are compatible with the renaming of free variables.

Example 2.5. The presheaves $F_{\lambda}, T_{\lambda}$ and $R_{\lambda}$ are algebras for $H_{\lambda}$ in the obvious sense.

\section{Remark 2.6.}

(i) The slice category $V /$ Set $^{\mathscr{F}}$ of presheaves $X$ together with a morphism $i: V \rightarrow X$ is called the category of pointed presheaves. For example $F_{\lambda}$ is a pointed presheaf in a canonical sense: $i^{F}: V \rightarrow F_{\lambda}$ takes a variable $x$ to the term $x$. Analogously $i^{T}: V \rightarrow T_{\lambda}$ and $i^{R}: V \rightarrow R_{\lambda}$ are pointed presheaves, and so are $F_{\lambda, \Sigma}, R_{\lambda, \Sigma}$ and $T_{\lambda, \Sigma}$.

(ii) Recall that the category $\mathbf{A l g} H_{\lambda}$ of algebras for $H_{\lambda}$ has as morphisms the usual $H_{\lambda^{-}}$ homomorphisms, i.e., a morphism from $a: H_{\lambda} X \rightarrow X$ to $b: H_{\lambda} Y \rightarrow Y$ is a natural 
transformation $f: X \rightarrow Y$ such that $f \cdot a=b \cdot H_{\lambda} f$. Then $\mathbf{A l g} H_{\lambda}$ is a concrete category over Set ${ }^{\mathscr{F}}$ with the forgetful functor $\left(H_{\lambda} X \rightarrow X\right) \mapsto X$.

Theorem 2.7 (see [10]). The presheaf $F_{\lambda}$ of finite $\lambda$-terms is the free $H_{\lambda}$-algebra on $V$.

Definition 2.8 (see [2]). Given an endofunctor $H$, an algebra $a: H A \rightarrow A$ is called

(1) completely iterative (cia for short) if for every object $X$ (of variables) and every (flat equation) morphism $e: X \rightarrow H X+A$ there exists a unique solution which means a unique morphism $e^{\dagger}: X \rightarrow A$ such that the square below commutes

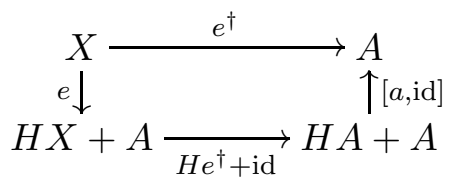

(2) iterative if every equation morphism $e: X \rightarrow H X+A$ with $X$ finitely presentable has a unique solution $e^{\dagger}: X \rightarrow A$.

We are going to characterize finitely presentable presheaves in Theorem 2.16. In practice, we are interested only in equations using free presheaves (on polynomial endofunctors of Set) as $X$, but including the more general concept does not "disturb" anything as we explain in Remark 5.2 .

Example 2.9. As proved in [17], Corollary 6.3, the free completely iterative algebra for an arbitrary finitary endofunctor $H$ on an object $X$ is precisely the terminal coalgebra for $H(-)+X$. More detailed, suppose $T X$ is the terminal coalgebra for $H(-)+X$, then its structure morphism is an isomorphism by Lambek's Lemma and the inverse of this morphism has the components

$$
\tau_{X}: H T X \rightarrow T X \quad \text { and } \quad \eta_{X}^{T}: X \rightarrow T X
$$

making $T X$ a free cia on $X$.

Conversely, let $\tau_{X}: H T X \rightarrow T X$ be a cia which is free on $X$ w.r.t. the universal arrow $\eta_{X}^{T}$. Then $\left[\tau_{X}, \eta_{X}^{T}\right]: H T X+X \rightarrow T X$ is an isomorphism, and its inverse is the structure of the terminal coalgebra for $H(-)+X$.

Theorem 2.10. The presheaf $T_{\lambda}$ of infinite $\lambda$-terms is the free completely iterative $H_{\lambda^{-}}$ algebra on $V$.

Proof. As explained in Example 2.9 above, the free completely iterative algebra for $H_{\lambda}$ on $V$ is precisely the terminal coalgebra for $H_{\lambda}(-)+V$. The latter functor clearly preserves limits of $\omega^{\mathrm{op}}$-chains. Consequently, its terminal coalgebra is a limit of the chain $W$ with $W_{0}=1$ (the terminal presheaf) and $W_{n+1}=H_{\lambda} W_{n}+V$, where the connecting maps are the unique $w_{0}: W_{1} \rightarrow W_{0}$ and $w_{n+1}=H_{\lambda} w_{n}+\mathrm{id}_{V}$.

Observe first that the limit of $W$ is computed objectwise. So for every context $\Gamma$ we can identify $W_{0}(\Gamma)$ with the set $\{\perp\}$ where $\perp \notin$ Var, and we have

$$
W_{n+1}(\Gamma)=W_{n}(\Gamma) \times W_{n}(\Gamma)+W_{n}(\Gamma+1)+\Gamma .
$$

An easy induction proof now shows that $W_{n}(\Gamma)$ can be identified with the set of all $\lambda$ terms in context $\Gamma$ of depth at most $n$ having all leaves of depth $n$ labelled by $\perp$. And $w_{n+1}: W_{n+1} \rightarrow W_{n}$ cuts away the level $n+1$ in the trees of $W_{n+1}(\Gamma)$, relabelling level- $n$ leaves by $\perp$. With this identification we obtain $T_{\lambda}$ as a limit of $W_{n}$ where the limit maps $T_{\lambda} \rightarrow W_{n}$ cut the trees in $T_{\lambda}(\Gamma)$ at level $n$ and relabel level- $n$ leaves by $\perp$. 
Example 2.11. The complete iterativity of the algebra $T_{\lambda}$ means that we are able to solve systems of recursive equations such as

$$
\begin{aligned}
& p_{1}=p_{1} @\left(\lambda x \cdot p_{2}\right) \\
& p_{2}=y @ p_{1} .
\end{aligned}
$$

Indeed, the solution in $T_{\lambda}(\{y\})$ is formed by the $\lambda$-terms represented by the following trees $\hat{t}_{1}$ and $\hat{t}_{2}$ :

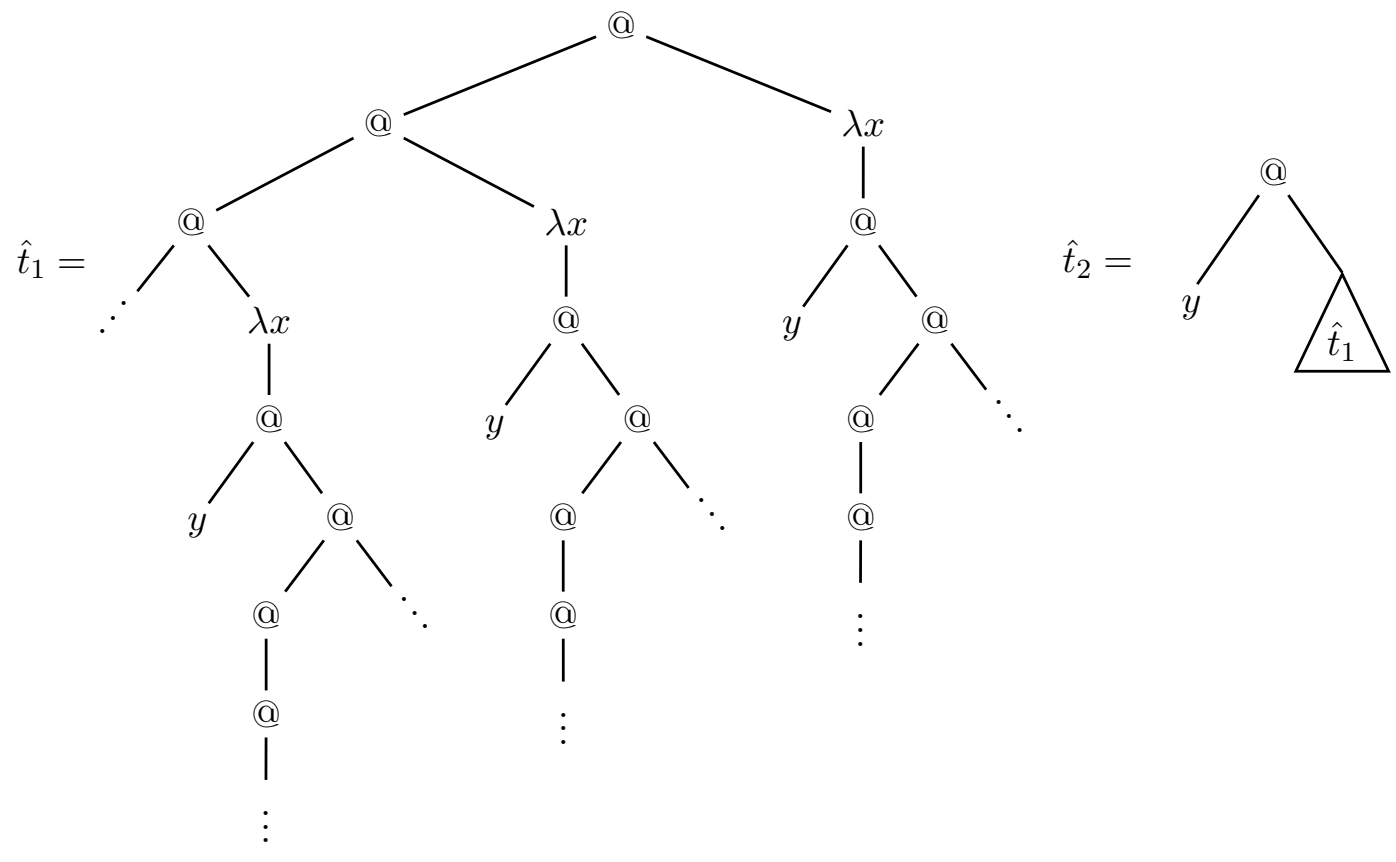

How is this related to the above concept of Definition 2.8? Firstly, every system of recursive equations can be flattened: a flat system has in context $\Gamma$ the right-hand sides of only three types: $p_{i} @ p_{j}$ or $\lambda x \cdot p_{i}$ or a term in $T_{\lambda}(\Gamma)$. For example, we flatten the system (2.4) to

$$
\begin{aligned}
& p_{1}=p_{1} @ p_{3} \\
& p_{2}=p_{4} @ p_{1} \\
& p_{3}=\lambda x \cdot p_{2} \\
& p_{4}=y
\end{aligned}
$$

Let $\Gamma=\{y\}$ be the context of all free variables and let $X$ be the free presheaf on generators $p_{1}, \ldots, p_{4}$ of context $\Gamma$, see Example 2.2. Notice that even though the recursion variables $p_{1}, \ldots, p_{4}$ appear as constants in the system (2.5), the associated presheaf $X$ is not a constant presheaf. Using the Yoneda lemma, the above system (2.5) defines an obvious morphism

$$
e: X \rightarrow H_{\lambda} X+T_{\lambda}
$$

viz, the unique one such that $e_{\Gamma}\left(p_{i}\right)$ is the right-hand side of the equation above. The solution

$$
e^{\dagger}: X \rightarrow T_{\lambda}
$$

is the unique morphism such that $e_{\Gamma}^{\dagger}$ takes $p_{i}$ to the solution in $T_{\lambda}$; for example $e_{\Gamma}^{\dagger}\left(p_{1}\right)=\left[\hat{t}_{1}\right]$ for the above tree $\hat{t}_{1}$. We will see in Theorem 5.7 below that equations such as (2.5) have a unique solution yielding rational trees. 
Remark 2.12. Given an equation morphism

$$
e: X \rightarrow H_{\lambda} X+T_{\lambda}
$$

then the solution $e^{\dagger}: X \rightarrow T_{\lambda}$ allows us to choose, for every element $p$ of $X(\Gamma)$, a tree $\hat{t}_{p}$ in $T_{\lambda}(\Gamma)$ with

$$
e_{\Gamma}^{\dagger}(p)=\left[\hat{t}_{p}\right]
$$

Due to the commutativity of (2.3) for $H_{\lambda} X=X \times X+\delta X$ we have three possible cases for every $p$ :

(a) $e_{\Gamma}(p)=\left(p_{1}, p_{2}\right)$ in $X(\Gamma) \times X(\Gamma)$, then for the operation $\tau: H_{\lambda} T_{\lambda} \rightarrow T_{\lambda}$ we have

$$
\left[\hat{t}_{p}\right]=\tau\left(\left[\hat{t}_{p_{1}}\right],\left[\hat{t}_{p_{2}}\right]\right)
$$

in other words,

(b) $e_{\Gamma}(p)=q$ in $X(\Gamma+\{x\})$, then

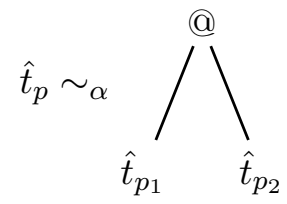

$$
\left[\hat{t}_{p}\right]=\tau\left(\left[\hat{t}_{q}\right]\right)
$$

in other words

$$
\left.\hat{t}_{q} \sim_{\alpha}\right|_{\hat{t}_{p}} ^{\lambda x}
$$

or

(c) $e_{\Gamma}(p)$ lies in $T_{\lambda}(\Gamma)$ and is represented by $\hat{t}_{p}$ :

$$
e_{\Gamma}^{\dagger}(p)=\left[\hat{t}_{p}\right]=e_{\Gamma}(p) .
$$

Remark 2.13. We are going to characterize the presheaf $R_{\lambda}$ as a free iterative algebra for $H_{\lambda}$. That is, in equations we admit only presheaves $X$ of variables that are finitely presentable. Recall that an object $X$ of a category $\mathscr{W}$ is finitely presentable provided that its hom-functor $\mathscr{W}(X,-)$ preserves filtered colimits. We are first going to characterize the finitely presentable presheaves by using the following concept:

Definition 2.14 (see [4]). A presheaf $X$ is called super-finitary provided that each $X(\Gamma)$ is finite and there exists a nonempty context $\Gamma_{0}$ generating $X$ in the sense that for every nonempty context $\Gamma$ we have

$$
X(\Gamma)=\bigcup_{\gamma: \Gamma_{0} \rightarrow \Gamma} X \gamma\left[X\left(\Gamma_{0}\right)\right]
$$

Example 2.15. A signature $\Sigma$ defines the polynomial presheaf $X_{\Sigma}$, see Example 2.2(ii), by $X_{\Sigma}(\Gamma)=\coprod_{\sigma \in \Sigma} \Gamma^{\operatorname{ar}(\sigma)}$. This is a super-finitary presheaf iff $\Sigma$ is a finite signature. Other super-finitary presheaves are precisely the quotients of $X_{\Sigma}$ with $\Sigma$ finite.

Theorem 2.16. A presheaf in Set $^{\mathscr{F}}$ is finitely presentable iff it is super-finitary. 
Proof. (1) Let $X$ be a super-finitary presheaf and let $\Gamma_{0}$ be a context of $n$ variables generating $X$. We prove that $X$ is a finite colimit of representables. Since representables are (due to Yoneda lemma) clearly finitely presentable, this proves finite presentability of $X$.

Form the finite diagram of all presheaves

$$
Z_{a}=\mathscr{F}(\Gamma,-)
$$

where $\Gamma \subseteq \Gamma_{0}+\Gamma_{0}$ is a context of at most $2 n$ variables 1 and $a \in X(\Gamma)$. The connecting morphisms are the Yoneda transformations

$$
Y f: Z_{a} \rightarrow Z_{a^{\prime}} \quad \text { for } a \in X(\Gamma) \text { and } a^{\prime} \in X\left(\Gamma^{\prime}\right)
$$

where $f: \Gamma^{\prime} \rightarrow \Gamma$ is a function that fulfils $X f\left(a^{\prime}\right)=a$. The Yoneda transformations

$$
z_{a}: Z_{a} \rightarrow X, \quad \text { with the components defined by } f \mapsto X f(a),
$$

clearly form a compatible cocone of this finite diagram. We prove that this is a colimit cocone. In other words, for every context $\bar{\Gamma}$ we must prove that the cocone of all $\bar{\Gamma}$ components $z_{a}^{\bar{\Gamma}}$ (sending elements $f: \Gamma \rightarrow \bar{\Gamma}$ of $Z_{a}=\mathscr{F}(\Gamma,-)$ to $\left.X f(a)\right)$ is a colimit in Set. For that we only need to verify that in every context $\bar{\Gamma}$

(i) the cocone $z_{a}^{\bar{\Gamma}}$ is collectively epimorphic, and

(ii) whenever two elements $f: \Gamma \rightarrow \bar{\Gamma}$ of $Z_{a}$ and $f^{\prime}: \Gamma^{\prime} \rightarrow \bar{\Gamma}$ of $Z_{a^{\prime}}$ fulfil $z_{a}^{\bar{\Gamma}}(f)=z_{a^{\prime}}^{\bar{\Gamma}_{1}}\left(f^{\prime}\right)$, then there exists a zig-zag connecting $f$ and $f^{\prime}$ in the $\bar{\Gamma}$-component of our diagram.

The proof of (i) is trivial: given an element $a \in X(\bar{\Gamma})$, either $\bar{\Gamma}=\emptyset$ or by Equation (2.6) there exists $f: \Gamma_{0} \rightarrow \bar{\Gamma}$ and an element $b \in X\left(\Gamma_{0}\right)$ with $a=X f(b)$, in other words,

$$
a=z_{b}^{\bar{\Gamma}}(f) \text {. }
$$

In case $\bar{\Gamma}=\emptyset$ we have $a=z_{a}^{\bar{\Gamma}}\left(\operatorname{id}_{\emptyset}\right)$.

To prove (ii), observe that the given equation states

$$
X f(a)=X f^{\prime}\left(a^{\prime}\right) \text {. }
$$

In case $\bar{\Gamma}$ has at most $2 n$ variables, we can assume $\bar{\Gamma} \subseteq \Gamma_{0}+\Gamma_{0}$ and the desired zig-zag is

$$
Z_{a} \stackrel{Y f}{\longleftarrow} Z_{b} \stackrel{Y f^{\prime}}{\longrightarrow} Z_{a^{\prime}}
$$

where $b=X f(a)$. Thus, we can assume that $\bar{\Gamma}$ has more than $2 n$ elements.

Case 1: $\Gamma=\emptyset=\Gamma^{\prime}$. Here $f=f^{\prime}$ and we have $X f(a)=X f\left(a^{\prime}\right)$. Choose a monomorphism $m: \Gamma_{0} \rightarrow \bar{\Gamma}$ and observe that $f=m \cdot g$ for the unique $g: \emptyset \rightarrow \Gamma_{0}$. Thus $X m(X g(a))=$ $X m\left(X g\left(a^{\prime}\right)\right)$ and since $m$ is a split monomorphism, we conclude $X g(a)=X g\left(a^{\prime}\right)=c$.

The desired zig-zag is

$$
Z_{a} \stackrel{Y g}{\longleftarrow} Z_{c} \stackrel{Y g^{\prime}}{\longrightarrow} Z_{a^{\prime}}
$$

Case 2: $\Gamma=\emptyset \neq \Gamma^{\prime}$. Factorize $f^{\prime}$ as an epimorphism $e$ followed by a split monomorphism $m$ :

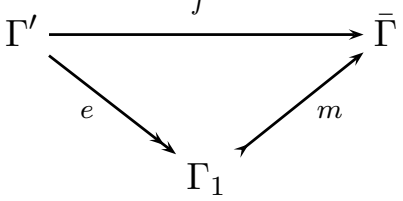

Then, since for the unique $h: \emptyset \rightarrow \Gamma_{1}$ we have $f=m \cdot h$, we obtain

$$
X m\left(X e\left(a^{\prime}\right)\right)=X m(X h(a)) .
$$

\footnotetext{
${ }^{1}$ The reason why we need $2 n$ variables will become clear in (2.7) below.
} 
Thus, $X e\left(a^{\prime}\right)=X h(a)=c$ which yields the zig-zag

$$
Z_{a} \stackrel{Y h}{\longleftarrow} Z_{c} \stackrel{Y e}{\longrightarrow} Z_{a^{\prime}}
$$

Case 3: $\Gamma \neq \emptyset \neq \Gamma^{\prime}$. Find $g: \Gamma_{0} \rightarrow \Gamma$ with $a=X g(b)$ and $g^{\prime}: \Gamma_{0} \rightarrow \Gamma^{\prime}$ with $a^{\prime}=X g^{\prime}\left(b^{\prime}\right)$ for some $b, b^{\prime} \in X\left(\Gamma_{0}\right)$. Then $X(f \cdot g)(b)=X\left(f^{\prime} \cdot g^{\prime}\right)\left(b^{\prime}\right)$. Now factorize $\left[f \cdot g, f^{\prime} \cdot g^{\prime}\right]: \Gamma_{0}+\Gamma_{0} \rightarrow$ $\bar{\Gamma}$ as an epimorphism followed by a split monomorphism; so we obtain a commutative diagram

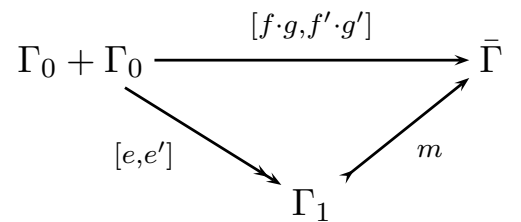

Since $m$ is a split monomorphism, conclude that $X e(b)=X e^{\prime}\left(b^{\prime}\right)=c$. The desired zig-zag is

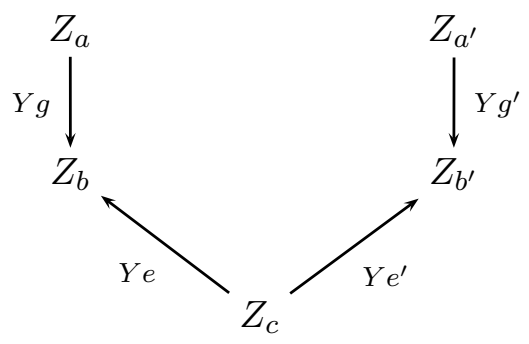

(2) Let $X$ be a finitely presentable object of Set $^{\mathscr{F}}$. The empty maps are denoted by $t_{\Gamma}: \emptyset \rightarrow \Gamma$. For every nonempty context $\Gamma_{0}$ let $X_{\Gamma_{0}}$ be the subfunctor of $X$ generated by the elements of $X\left(\Gamma_{0}\right) \cup X(\emptyset)$ : it assigns to every $\Gamma$ the subset of $X(\Gamma)$ given by

$$
X_{\Gamma_{0}}(\Gamma)=X t_{\Gamma}[X(\emptyset)] \cup \underset{f: \Gamma_{0} \rightarrow \Gamma}{\bigcup} X f\left[X\left(\Gamma_{0}\right)\right]
$$

We obviously have a union

$$
X=\bigcup_{\Gamma_{0} \in \mathscr{F} \backslash\{\emptyset\}} X_{\Gamma_{0}}
$$

which is directed: given nonempty contexts $\Gamma_{0}, \Gamma_{1}$ then $X_{\Gamma_{0}} \cup X_{\Gamma_{1}} \subseteq X_{\Gamma_{0} \cup \Gamma_{1}}$. Since $X$ is finitely presentable, the morphism

$$
\operatorname{id}_{X}: X \rightarrow \operatorname{colim}_{\Gamma_{0} \in \mathscr{F} \backslash\{\emptyset\}} X_{\Gamma_{0}}
$$

factorizes through one of the colimit injections $X_{\Gamma_{0}} \hookrightarrow X$. In other words

$$
X=X_{\Gamma_{0}} \quad \text { for some } \Gamma_{0} \neq \emptyset \text {. }
$$

It remains to prove that the sets $X\left(\Gamma_{0}\right)$ and $X(\emptyset)$ are finite, then every $X(\Gamma)$ is finite.

For every finite set $M \subseteq X(\emptyset)$ we have the subfunctor $X^{M}$ of $X$ equal to $X$ on nonempty objects and maps, and assigning $M$ to $\emptyset$. We obviously get $X$ as a directed union of these subfunctors $X^{M}$, thus, as above, there exists $M$ with $X=X^{M}$. Then $X(\emptyset)=M$ is finite.

For every finite set $M \subseteq X\left(\Gamma_{0}\right)$ we have the subfunctor ${ }^{M} X$ of $X=X_{\Gamma_{0}}$ generated by the elements of $M \cup X(\emptyset)$ :

$$
{ }^{M} X(\Gamma)=X t_{\Gamma}[X(\emptyset)] \cup \underset{f: \Gamma_{0} \rightarrow \Gamma}{\bigcup} X f[M] .
$$


Again $X$ is a directed union of these subfunctors ${ }^{M} X$, thus, there exists $M$ with $X={ }^{M} X$, proving that $X\left(\Gamma_{0}\right)$ is finite.

Theorem 2.17. The presheaf $R_{\lambda}$ of rational $\lambda$-terms is the free iterative $H_{\lambda}$-algebra on $V$.

Proof. (I) $R_{\lambda}$ is an iterative algebra for $H_{\lambda}$. Indeed, given an equation morphism

$$
e: X \rightarrow H_{\lambda} X+R_{\lambda}
$$

where Equation (2.6) holds for $\Gamma_{0}$, we know that its extension

$$
\bar{e}: X \stackrel{e}{\longrightarrow} H_{\lambda} X+R_{\lambda} \hookrightarrow H_{\lambda} X+T_{\lambda}
$$

has a unique solution $e^{\dagger}: X \rightarrow T_{\lambda}$, and we are going to prove that the trees $e_{\Gamma_{0}}^{\dagger}(p)$ and $e_{\emptyset}^{\dagger}(p)$ are all rational. It then follows that all the trees $e_{\Gamma}^{\dagger}(p)$ are rational for all contexts $\Gamma$, and this gives us the desired solution $X \rightarrow R_{\lambda}$. Indeed, for each $x \in X(\Gamma)$ with $\Gamma \neq \emptyset$ we have $x=X f(p)$ for some $f: \Gamma_{0} \rightarrow \Gamma$ and $p \in X\left(\Gamma_{0}\right)$. Then $e_{\Gamma}^{\dagger}(x)=e_{\Gamma}^{\dagger}(X f(p))=T_{\lambda} f\left(e_{\Gamma_{0}}^{\dagger}(p)\right)$ by the naturality of $e^{\dagger}$, and since $e_{\Gamma_{0}}^{\dagger}(p)$ is rational, so is $T_{\lambda} f\left(e_{\Gamma_{0}}^{\dagger}(p)\right)$. (The action of $T_{\lambda} f$ is just relabelling leaves according to $f$.)

Now every element of $X\left(\Gamma_{0}\right)=\left\{p_{1}, \ldots, p_{n}\right\}$ yields an element

$$
e_{\Gamma_{0}}\left(p_{i}\right) \in X\left(\Gamma_{0}\right) \times X\left(\Gamma_{0}\right)+X\left(\Gamma_{0}+\{x\}\right)+R_{\lambda}\left(\Gamma_{0}\right)
$$

which is either (i) a pair $\left(p_{j}, p_{k}\right)$ or (ii) $q \in X\left(\Gamma_{0}+\{x\}\right)$ or (iii) a rational tree in $R_{\lambda}\left(\Gamma_{0}\right)$. Put $t_{i}=e_{\Gamma_{0}}^{\dagger}\left(p_{i}\right)$, then in the last case the commutativity of Diagram (2.3) implies that $e_{\Gamma_{0}}\left(p_{i}\right)=t_{i}$ (cf. Remark 2.12). From (2.3) we also obtain in cases (i) and (ii)

$$
t_{i}=t_{j} @ t_{k} \quad \text { and } \quad t_{i}=\lambda x \cdot e_{\Gamma_{0}+\{x\}}^{\dagger}(q), \quad \text { respectively. }
$$

From Equation (2.6) we see that in case (ii) there exists $f: \Gamma_{0} \rightarrow \Gamma_{0}+\{x\}$ with $q=X f\left(p_{j}\right)$ for some $j$, then $e_{\Gamma_{0}+\{x\}}^{\dagger}(q)=T_{\lambda} f\left(e_{\Gamma_{0}}^{\dagger}\left(p_{j}\right)\right)=T_{\lambda} f\left(t_{j}\right)$. Thus we get equations telling us that for every $i$ either $t_{i}=t_{j} @ t_{k}$ or $t_{i}=\lambda x \cdot T_{\lambda} f\left(t_{j}\right)$ or $t_{i}$ is a rational tree. Using these equations it is now easy, for every $i=1, \ldots, n$, to prove by induction on the depth $k$ of subtrees of $t_{i}$ that each subtree of $t_{i}$ is either of the form $s=T_{\lambda} f\left(e_{\Gamma_{0}}^{\dagger}(r)\right)$ for some $r \in X\left(\Gamma_{0}\right)$ and some $f: \Gamma_{0} \rightarrow \Gamma_{0}+\{x\}$, or $s$ is a subtree of some rational tree $e_{\Gamma_{0}}^{\dagger}(r)=e_{\Gamma_{0}}(r)$ in case (iii). Since $X\left(\Gamma_{0}\right)$ is a finite set, it follows that every tree $t_{i}$ has only finitely many subtrees, whence $t_{i} \in R_{\lambda}\left(\Gamma_{0}\right)$.

The case $X(\emptyset)=\left\{p_{1}, \ldots, p_{n}\right\}$ is analogous: for $t_{i}=e_{\Gamma}^{\dagger}\left(p_{i}\right)$ we get (i) $t_{i}=t_{j} @ t_{k}$ or (ii) $t_{i}=\lambda x \cdot e_{\{x\}}^{\dagger}(q)$ or (iii) $t_{i}=e_{\emptyset}\left(p_{i}\right) \in R_{\lambda}(\emptyset)$. We already know that the trees in case (ii) are rational. Thus, each subtree of $e_{\emptyset}^{\dagger}\left(p_{i}\right)$ is either $e_{\emptyset}^{\dagger}(r)$ or it is a subtree of some rational tree in cases (ii) or (iii).

The solution of $e$ in $R_{\lambda}$ is unique because every solution in $R_{\lambda}$ yields a solution of the extended morphism $\bar{e}$ in $T_{\lambda}$.

(II) Let $\mathscr{D}$ be the category of all equation morphisms

$$
e: X \rightarrow H_{\lambda} X+V, \quad X \text { finitely presentable, }
$$

whose morphisms are the coalgebra homomorphisms for $H_{\lambda}(-)+V$. The diagram $D: \mathscr{D} \rightarrow$ Set $^{\mathscr{F}}, D(e)=X$, is filtered and its colimit is the free iterative $H_{\lambda}$-algebra on $V$, see [2]. We will prove that $R_{\lambda}$ is a colimit of $D$. Recall that $R_{\lambda}$ is a pointed presheaf (see Remark 2.6). 
For every $e$ as above the equation morphism

$$
\tilde{e} \equiv X \stackrel{e}{\longrightarrow} H_{\lambda} X+V \stackrel{\mathrm{id}+i^{R}}{\longrightarrow} H_{\lambda} X+R_{\lambda}
$$

has a unique solution $\tilde{e}^{\dagger}: X \rightarrow R_{\lambda}$. It is easy to verify that these morphisms form a cocone for the diagram $D$. Since $D$ is a filtered diagram in Set $^{\mathscr{F}}$ and since colimits in Set $^{\mathscr{F}}$ are constructed objectwise in Set, in order to prove that

$$
R_{\lambda}=\operatorname{colim} D \quad \text { with the colimit cocone }\left(\tilde{e}^{\dagger}\right)
$$

all we need to prove is that for every context $\Gamma$

(a) the cocone $\tilde{e}_{\Gamma}^{\dagger}$ is collectively epimorphic: $R_{\lambda}(\Gamma)=\bigcup \tilde{e}_{\Gamma}^{\dagger}[X]$, and

(b) whenever $\tilde{e}_{\Gamma}^{\dagger}$ merges $x, x^{\prime} \in X(\Gamma)$, there exists a connecting morphism in $\mathscr{D}$ merging $x$ and $x^{\prime}$ too.

To prove (a), let $t \in R_{\lambda}(\Gamma)$ be a rational tree and let $\Gamma_{0}$ be the context of variables $x_{s}$ indexed by the finitely many subtrees $s$ of $t$ (up to isomorphism). Let $X$ be the free presheaf on the set $\Gamma_{0}$ of generators of context $\bar{\Gamma}=\Gamma \cup \Gamma_{0}$, see Example 2.2(ii). Define

$$
e: X \rightarrow H_{\lambda} X+V
$$

by assigning to every variable $x_{s}$, for a subtree $s$ of $t$, the following value: if $s=s^{\prime} @ s^{\prime \prime}$ in $t$, then

$$
e_{\Gamma}\left(x_{s}\right)=x_{s^{\prime}} @ x_{s^{\prime \prime}} \quad \text { in } X(\bar{\Gamma}) \times X(\bar{\Gamma}),
$$

if $s=\lambda y \cdot s^{\prime}$ in $t$, then

$$
e_{\Gamma}\left(x_{s}\right)=\lambda y \cdot x_{s^{\prime}} \quad \text { in } X(\bar{\Gamma}+\{y\})
$$

and if $s$ is a leaf labelled by $x \in \Gamma$, then

$$
e_{\Gamma}\left(x_{s}\right)=x \quad \text { in } \Gamma=V(\Gamma) .
$$

This object $e$ of $\mathscr{D}$ yields two equation morphisms: $\tilde{e}: X \rightarrow H_{\lambda} X+R_{\lambda}$ above, and analogously $\hat{e}=\left(\mathrm{id}+i^{T}\right) \cdot e: X \rightarrow H_{\lambda} X+T_{\lambda}$. The solution of the latter is the unique morphism

$$
\hat{e}^{\dagger}: X \rightarrow T_{\lambda} \quad \text { with } \quad \hat{e}_{\bar{\Gamma}}^{\dagger}\left(x_{s}\right)=s \text { for all } s \in \Gamma_{0} .
$$

Indeed, Diagram (2.3) is easily seen to commute for $\hat{e}$ and $\hat{e}^{\dagger}$. In (I) above we saw that the solution $\tilde{e}^{\dagger}: X \rightarrow R_{\lambda}$ is a codomain restriction of $\hat{e}^{\dagger}$. In particular:

$$
t=\tilde{e}_{\bar{\Gamma}}^{\dagger}\left(x_{t}\right) .
$$

This proves (a).

To prove (b) let $\tau: H_{\lambda} T_{\lambda} \rightarrow T_{\lambda}$ denote the algebra structure of $T_{\lambda}$. By Theorem 2.10 and Example 2.9 we have that

$$
\left[\tau, i^{T}\right]: H_{\lambda} T_{\lambda}+V \rightarrow T_{\lambda} \quad \text { is an isomorphism. }
$$

From Diagram (2.3) we get

$$
\hat{e}^{\dagger}=\left[\tau, \mathrm{id}_{T_{\lambda}}\right] \cdot\left[H_{\lambda} \hat{e}^{\dagger}+\operatorname{id}_{T_{\lambda}}\right] \cdot\left(\operatorname{id}_{H_{\lambda} X}+i^{T}\right) \cdot e
$$

which yields

$$
\left[\tau, i^{T}\right]^{-1} \cdot \hat{e}^{\dagger}=\left(H_{\lambda} \hat{e}^{\dagger}+\operatorname{id}_{V}\right) \cdot e .
$$

Let us factorize $\hat{e}^{\dagger}$ as a strong epimorphism $k: X \rightarrow Y$ followed by a monomorphism $m: Y \rightarrow T_{\lambda}$. Then the last equation makes it possible to apply the diagonal fill in: 


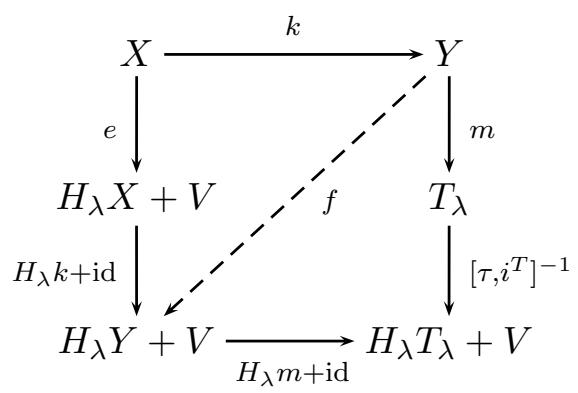

Indeed, $H_{\lambda}=(-)^{2}+\delta$ preserves connected limits (because each summand does), thus, monomorphisms; consequently, $H_{\lambda} m+\mathrm{id}_{V}$ is a monomorphism. Since $Y$ is a strong quotient of $X$, it follows from Theorem 2.16 that $Y$ is finitely presentable. Thus,

$$
f: Y \rightarrow H_{\lambda} Y+V
$$

is an object of $\mathscr{D}$, and clearly $k$ is a connecting morphism from $e$ to $f$.

From (I) we know that $\tilde{e}^{\dagger}$ is the domain restriction of $\hat{e}^{\dagger}$, thus we see that $\tilde{e}_{\Gamma}^{\dagger}(x)=\tilde{e}_{\Gamma}^{\dagger}\left(x^{\prime}\right)$ implies $\hat{e}_{\Gamma}^{\dagger}(x)=\hat{e}_{\Gamma}^{\dagger}\left(x^{\prime}\right)$, and since $m_{\Gamma}$ is a monomorphism with $\hat{e}_{\Gamma}^{\dagger}=m_{\Gamma} \cdot k_{\Gamma}$, we conclude

$$
k_{\Gamma}(x)=k_{\Gamma}\left(x^{\prime}\right)
$$

as requested.

Remark 2.18. As mentioned in the Introduction we want to combine application and abstraction with other operations. Suppose $\Sigma=\left(\Sigma_{n}\right)_{n \in \mathbb{N}}$ is a signature (of "terminals"). Then we can form the endofunctor $H_{\lambda, \Sigma}$ of Set $^{\mathscr{F}}$ on objects by

$$
H_{\lambda, \Sigma} X=X \times X+\delta X+\coprod_{n \in \mathbb{N}} \Sigma_{n} \bullet X^{n}
$$

where $\Sigma_{n} \bullet X^{n}$ is the coproduct (that is: disjoint union in every context) of $\Sigma_{n}$ copies of the $n$-th Cartesian power of $X$. For this endofunctor an algebra is an $H_{\lambda}$-algebra $A$ together with an $n$-ary operation on $A(\Gamma)$ for every $\sigma \in \Sigma_{n}$ and every context $\Gamma$.

In the following result we use notation of Example 2.2(vii).

Theorem 2.19. For every signature $\Sigma$

(i) $F_{\lambda, \Sigma}$ is the free $H_{\lambda, \Sigma}$-algebra on $V$,

(ii) $R_{\lambda, \Sigma}$ is the free iterative $H_{\lambda, \Sigma}$-algebra on $V$, and

(iii) $T_{\lambda, \Sigma}$ is the free completely iterative $H_{\lambda, \Sigma}$-algebra on $V$.

Indeed, (i) was proved in [10], and the proofs of (ii) and (iii) are completely analogous to the proofs of Theorems 2.17 and 2.10 .

\section{Presheaves as Monoids}

So far we have not treated one of the basic features of $\lambda$-calculus: substitution of subterms. For the presheaf $F_{\lambda, \Sigma}$ of finite $\lambda$ - $\Sigma$-terms this was elegantly performed by Fiore et al [10] based on the monoidal structure of the category Set ${ }^{\mathscr{F}}$. As mentioned in Notation 2.1(3), we can work with the equivalent category Fin(Set, Set) of all finitary endofunctors of Set. Composition of functors makes this a (strict, non-symmetric) monoidal 
category with unit $\operatorname{Id}_{\text {Set }}$. This monoidal structure, as shown in [10], corresponds to simultaneous substitution. Indeed, let $X$ and $Y$ be objects of Fin(Set, Set). Then the "formulas of the composite presheaf $X \cdot Y$ " in context $\Gamma$ are the elements of

$$
X \cdot Y(\Gamma)=X(Y(\Gamma))=\bigcup_{u: \bar{\Gamma} \hookrightarrow Y(\Gamma)} X u[\bar{\Gamma}]
$$

where $u: \bar{\Gamma} \hookrightarrow Y(\Gamma)$ ranges over finite subobjects of $Y(\Gamma)$. Indeed, $X$ preserves the filtered colimit $Y(\Gamma)=\operatorname{colim} \bar{\Gamma}$.

Consequently, in order to specify an $X \cdot Y$-formula $t$ in context $\Gamma$ we need (a) an $X$ formula $s$ in some new context $\bar{\Gamma}$ and (b) for every variable $x \in \bar{\Gamma}$ a $Y$-formula of context $\Gamma$, say, $r_{x}$. We can then think of $t$ as the formula $s\left(r_{x} / x\right)$ obtained from $s$ by simultaneous substitution.

Example 3.1. We consider the presheaves $F_{\lambda, \Sigma}$ and $F_{\lambda, \Sigma^{\prime}}$, where $\Sigma$ is the signature with a binary operation symbol $*$ and $\Sigma^{\prime}$ a signature with a unary operation symbol $o$. Then for every context $\Gamma$, the elements of $F_{\lambda, \Sigma} \cdot F_{\lambda, \Sigma^{\prime}}(\Gamma)$ are $\lambda$ - $\Sigma$-terms in some context $\bar{\Gamma}$ with free variables replaced by $\lambda$ - $\Sigma^{\prime}$-terms in context $\Gamma$. For a concrete example, let $\bar{\Gamma}=\{y, z\}$ and $\Gamma=\left\{z^{\prime}\right\}$ and consider the $\lambda$ - $\Sigma$-term

$$
t=\lambda x \cdot x *(y * z) \quad \text { in } F_{\lambda, \Sigma}\{y, z\}
$$

and the function

$$
u: \bar{\Gamma} \rightarrow F_{\lambda, \Sigma^{\prime}}(\Gamma) \quad \text { with } \begin{array}{r}
u(y)=\lambda x . o(x) @ z^{\prime} \\
u(z)=z^{\prime} @ o\left(o\left(z^{\prime}\right)\right)
\end{array}
$$

Then the element of $F_{\lambda, \Sigma} \cdot F_{\lambda, \Sigma^{\prime}}(\Gamma)$ corresponding to $t$ and $u$ is the term

$$
\lambda x . x *\left(\left(\lambda x . o(x) @ z^{\prime}\right) *\left(z^{\prime} @ o\left(o\left(z^{\prime}\right)\right)\right)\right) .
$$

\section{Remark 3.2.}

(i) The monoidal structure on Set $^{\mathscr{F}}$ corresponding to composition in Fin(Set, Set) will be denoted by $\otimes$. Its unit (corresponding to Id) is $V$, see Notation $2.2(\mathrm{i})$. Observe that every endofunctor $-\otimes X$ preserves colimits, e.g., $(A+B) \otimes X \cong(A \otimes X)+(B \otimes X)$.

(ii) Explicitly, the monoidal structure can be described by the coend

$$
(X \otimes Y)(\Gamma)=\int^{\bar{\Gamma}} \operatorname{Set}(\bar{\Gamma}, Y(\Gamma)) \bullet X(\bar{\Gamma}) .
$$

(iii) Recall that monoids in the monoidal category Fin(Set, Set) are precisely the finitary monads on Set.

(iv) The presheaf $F_{\lambda, \Sigma}$ is endowed with the usual simultaneous substitution of $\lambda$-terms which defines a morphism $m^{F}: F_{\lambda, \Sigma} \otimes F_{\lambda, \Sigma} \rightarrow F_{\lambda, \Sigma}$. Together with the canonical pointing $i^{F}: V \rightarrow F_{\lambda, \Sigma}$, see Remark [2.6, this constitutes a monoid as proved in [10].

Analogously the simultaneous substitution of infinite $\lambda$-terms defines a monoid

$$
\left(T_{\lambda, \Sigma}, m^{T}, i^{T}\right)
$$

It is easy to see that given a rational term, every simultaneous substitution of rational terms for variables yields again a rational term. Thus, we have a submonoid $\left(R_{\lambda, \Sigma}, m^{R}, i^{R}\right)$. 
(v) The monoidal operation of $F_{\lambda, \Sigma}$ is well connected to its structure of an $H_{\lambda, \Sigma}$-algebra. This was expressed in [10] by the concept of an $H_{\lambda, \Sigma}$-monoid.

In order to recall this concept, we need the notion of point-strength introduced in [9] under the name $(I / \mathscr{W})$-strength; this is a weakening of the classical strength (necessary since $H_{\lambda, \Sigma}$ is unfortunately not strong). Recall that given an object $I$ of a category $\mathscr{W}$, then objects of the slice category $I / \mathscr{W}$ are morphisms $x: I \rightarrow X$ for $X \in \operatorname{obj} \mathscr{W}$.

Definition 3.3 (see [9]). Let $(\mathscr{W}, \otimes, I)$ be a strict monoidal category and $H$ an endofunctor on $\mathscr{W}$. A point-strength of $H$ is a collection of morphisms

$$
s_{(X, x)(Y, y)}: H X \otimes Y \rightarrow H(X \otimes Y)
$$

natural in $(X, x)$ and $(Y, y)$ ranging through $I / \mathscr{W}$ such that

(i) $s_{(X, x)(I, \text { id })}=\operatorname{id}_{H X}$, and

(ii) the following triangles commute:

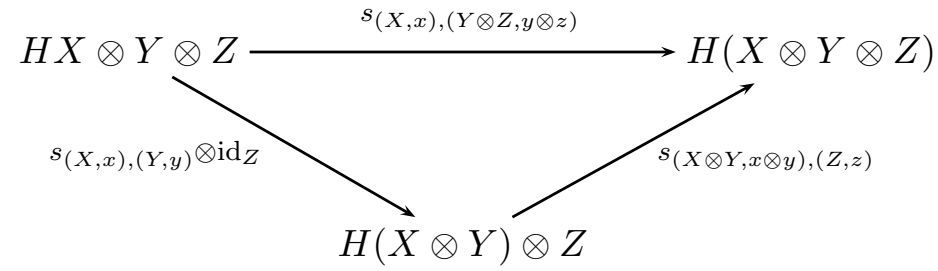

\section{Example 3.4.}

(i) The endofunctor $X \mapsto X \otimes X$ (which usually fails to be strong) has the point-strength

$$
s_{(X, x)(Y, y)}=(X \otimes X) \otimes Y=(X \otimes I \otimes X) \otimes Y \stackrel{\operatorname{id}_{X} \otimes y \otimes \operatorname{id}_{X \otimes Y}}{\longrightarrow}(X \otimes Y) \otimes(X \otimes Y) .
$$

(ii) The endofunctor $X \mapsto X^{n}$ of Set ${ }^{\mathscr{F}}$ is clearly (point-)strong for every $n \in \mathbb{N}$.

(iii) The functor $\delta$ in Notation 2.3 is point-strong, as observed in [10]. The easiest way to describe its point-strength is by working in Fin(Set, Set). Given pointed endofunctors $x:$ Id $\rightarrow X$ and $y:$ Id $\rightarrow Y$, then the point-strength $s_{(X, x)(Y, y)}:(\delta X) \cdot Y \rightarrow \delta(X \cdot Y)$ has components

$$
X(Y(\Gamma)+1) \stackrel{X\left(\mathrm{id}+y_{1}\right)}{\longrightarrow} X(Y(\Gamma)+Y(1)) \stackrel{X \text { can }}{\longrightarrow} X \cdot Y(\Gamma+1),
$$

where can: $Y(\Gamma)+Y(1) \rightarrow Y(\Gamma+1)$ denotes the canonical morphism.

(iv) A coproduct of point-strong functors is point-strong.

Corollary 3.5. The endofunctors $H_{\lambda}$ and $H_{\lambda, \Sigma}$ are point-strong. Their point-strength is denoted by $s^{H}$.

Definition 3.6 (see [10]). Let $H$ be a point-strong endofunctor of a monoidal category. By an $H$-monoid is meant an $H$-algebra $(A, a)$ which is also a monoid

$$
m: A \otimes A \rightarrow A \quad \text { and } \quad i: I \rightarrow A
$$

such that the square below commutes:

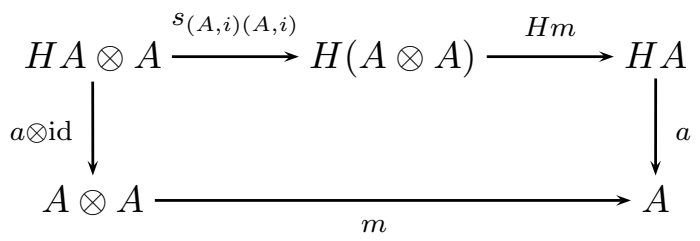




\section{Remark 3.7.}

(1) Homomorphisms of $H$-monoids are those monoid homomorphisms which are also $H$ algebra homomorphisms.

(2) An $H$-monoid is called (completely) iterative if its underlying $H$-algebra has this property.

\section{Example 3.8.}

(1) $F_{\lambda}$ is an $H_{\lambda}$-monoid. Indeed, we know that substitution yields the monoid structure (Remark 2.6) and tree tupling yields the algebra structure (Example 2.5). Let us consider the square

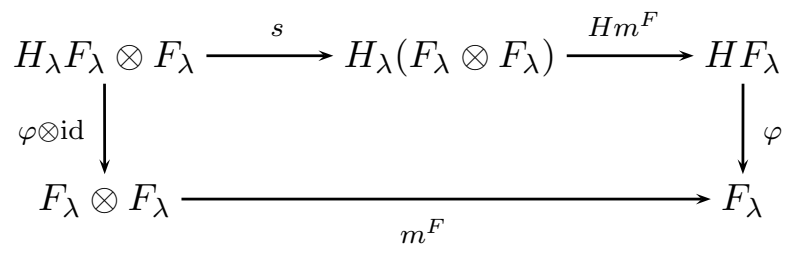

The elements $t$ of $H_{\lambda} F \otimes F_{\lambda}$ in context $\Gamma$ are those of

$$
H_{\lambda} F_{\lambda}\left(\Gamma_{0}\right)=F_{\lambda}\left(\Gamma_{0}\right) \times F_{\lambda}\left(\Gamma_{0}\right)+F_{\lambda}\left(\Gamma_{0}+\{x\}\right)
$$

for a given context $\Gamma_{0}$ together with a substitution $f: \Gamma_{0} \rightarrow F_{\lambda}(\Gamma)$. In case of the summand $F_{\lambda}\left(\Gamma_{0}\right) \times F_{\lambda}\left(\Gamma_{0}\right)$ the lower passage $m_{\Gamma}^{F}\left(\varphi_{\Gamma} \otimes \mathrm{id}\right)$ assigns to $t=\left(t_{1}, t_{2}\right)$ the term $t_{1} @ t_{2}$ with variables substituted according to $f$. And the upper passage first substitutes to $t_{1}$ and $t_{2}$ according to $f$ separately, and then forms @; the result is the same. In case of the summand $F_{\lambda}\left(\Gamma_{0}+\{x\}\right)$ the lower passage assigns to $t$ the term $\lambda x$.t with variables substituted according to $f$; the upper one first substitutes in $t$ and then forms $\lambda x$. - yielding the same result again.

(2) More generally, for every signature $\Sigma$ we have an $H_{\lambda, \Sigma}$-monoid $F_{\lambda, \Sigma}$.

Theorem 3.9 (see [10]). The presheaf $F_{\lambda, \Sigma}$ of finite $\lambda$ - $\Sigma$-terms is the initial $H_{\lambda, \Sigma}$-monoid.

Theorem 3.10 (see [16]). The presheaf $T_{\lambda, \Sigma}$ of $\lambda$ - $\Sigma$-terms is an $H_{\lambda, \Sigma}$-monoid with simultaneous substitution as monoid structure.

Although in [16], Example 13, just $T_{\lambda}$ is used, the methods of that paper apply to $T_{\lambda, \Sigma}$ immediately. The following theorem proves a stronger property of $T_{\lambda, \Sigma}$, corresponding to Theorem 3.9 above.

Theorem 3.11. The presheaf $T_{\lambda, \Sigma}$ of $\lambda$ - $\Sigma$-terms is the initial completely iterative $H_{\lambda, \Sigma^{-}}$ monoid.

An elementary proof of this theorem was presented in [4]. Here we will prove a more general result in Theorem 4.18 below. 


\section{The Initial Iterative $H$-Monoid}

The aim of this section is to prove that the presheaf $R_{\lambda, \Sigma}$ of rational $\lambda$ - $\Sigma$-terms is the initial iterative $H_{\lambda, \Sigma}$-monoid in $\mathbf{S e t}^{\mathscr{F}}$. We have (in contrast to the characterization of $T_{\lambda}$ in the preceding section) no elementary proof. Rather, we need to work with the monad $\mathbb{R}_{\lambda, \Sigma}$ of free iterative $H_{\lambda, \Sigma}$-algebras on Set $^{\mathscr{F}}$ (for which $R_{\lambda, \Sigma}$ is $\mathbb{R}_{\lambda, \Sigma}(V)$ ) and prove that it is point-strong and use this strength further. We will actually work in a more general setting (which can be applied later for the case of typed $\lambda$-calculus).

Assumption 4.1. Throughout this section we assume that $H$ is a finitary endofunctor of $\mathscr{W}$ where

(1) $\mathscr{W}$ is a locally finitely presentable category, i.e., a cocomplete category with a set of finitely presentable objects $\mathscr{W}_{\text {fp }}$ whose closure under filtered colimits is all of $\mathscr{W}$.

(2) $\mathscr{W}$ is also a strict monoidal category with the unit $I$ finitely presentable and the tensor product preserving finite presentability: if $A, B$ are finitely presentable, then so is $A \otimes B$.

(3) $\mathscr{W}$ is right distributive, that is, for every object $W$ the endofunctor $-\otimes W$ preserves finite coproducts.

(4) The tensor product is a finitary functor, i.e., its preserves filtered colimits (in both variables).

We call categories satisfying (1)-(4) monoidally locally finitely presentable.

Example 4.2. Set is, as a cartesian closed category, monoidally locally finitely presentable. For every monoidally locally finitely presentable category $\mathscr{W}$ all functor categories $\mathscr{W}^{\mathscr{A}}$, $\mathscr{A}$ small, have the property too; for example, Set $^{\mathscr{F}}$ with the cartesian product as tensor. However, in our paper we only use the fact that Set $^{\mathscr{F}}$ is a monoidally locally finitely presentable w.r.t. $\otimes$ in Remark 3.2(i). This follows from the fact that this is equivalent to Fin(Set, Set) with the tensor product given by composition. Observe that $\otimes$ is right distributive (since precomposition with a given functor preserves colimits) but not left distributive.

Notation 4.3. For every object $Y$ of $\mathscr{W}$ we denote by

$$
\varrho_{Y}: H R Y \rightarrow R Y \quad \text { and } \quad \eta_{Y}: Y \rightarrow R Y
$$

the structure and oniversal morphism of the free iterative $H$-algebra on $Y$, which exists as proved in [2]. This gives rise to the monad

$$
\mathbb{R}=(R, \eta, \mu)
$$

where $\mu_{X}: R R Y \rightarrow R Y$ is the unique homomorphism extending $\eta_{Y}$ :

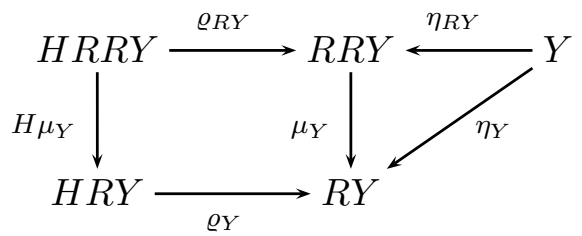

$\mathbb{R}$ is called the rational monad of the endofunctor $H$.

Remark 4.4. In [2] we described the free iterative $H$-algebra $R Y$ as the colimit of the diagram of all "flat equation" morphisms

$$
e: W \rightarrow H W+Y, \quad W \in \mathscr{W} \text { finitely presentable, }
$$


whose connecting morphisms ("equation morphisms") are just the coalgebra homomorphisms $h$ for the endofunctor $H(-)+Y$ :

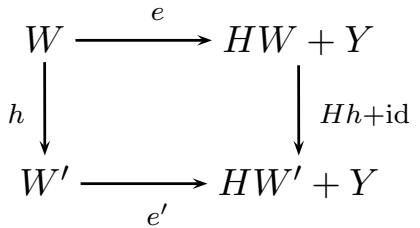

More detailed:

(i) The category $\mathrm{EQ}_{Y}$ of all flat equation morphisms in $Y$ is filtered. The filtered diagram

$$
\mathrm{Eq}_{Y}: \mathrm{EQ}_{Y} \rightarrow W, \quad \mathrm{Eq}_{Y}(W \stackrel{e}{\rightarrow} H W+Y)=W
$$

has a colimit $R Y$ with the colimit injections $e^{\#}: W \rightarrow R Y$.

(ii) For the flat equation morphism inl: $Y \rightarrow H Y+Y$ put

$$
\eta_{Y}=\operatorname{inr}^{\#}: Y \rightarrow R Y \text {. }
$$

(iii) There is a unique isomorphism $i: R Y \rightarrow H R Y+Y$ such that the squares

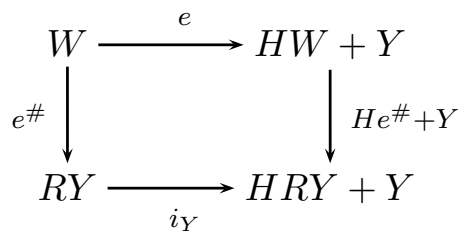

commute for all flat equations $e$. Put

$$
\varrho \equiv H R Y \stackrel{\text { inl }}{\longrightarrow} H R Y+Y \stackrel{i_{Y}^{-1}}{\longrightarrow} R Y .
$$

Then $R Y$ together with $\eta_{Y}$ and $\varrho_{Y}$ is the free iterative $H$-algebra on $Y$. We also have

$$
i_{Y}=\left[\varrho_{Y}, \eta_{Y}\right]^{-1} \text {. }
$$

Furthermore, $e^{\#}$ is the unique coalgebra homomorphism from $e$ to $i_{Y}$.

(iv) For every $e: W \rightarrow H W+Y$ the morphism $e^{\#}: W \rightarrow R Y$ is the unique solution (in the iterative algebra $R Y$ ) of

$$
\eta_{Y} \bullet e \equiv W \stackrel{e}{\longrightarrow} H W+Y \stackrel{H W+\eta_{Y}}{\longrightarrow} H W+R Y .
$$

(v) Let

$$
e: W \rightarrow H W+R Y \quad \text { and } \quad e^{\prime}: W^{\prime} \rightarrow H W^{\prime}+R Y
$$

be two equation morphisms with $W$ and $W^{\prime}$ finitely presentable, and let $h$ be a coalgebra homomorphism from $(W, e)$ to $\left(W, e^{\prime}\right)$. Then for the unique solutions of $e$ and $e^{\prime}$ we have

$$
e^{\dagger}=\left(e^{\prime}\right)^{\dagger} \cdot h: W \rightarrow R Y .
$$

(v) Suppose we have two morphisms

$$
f: V \rightarrow H V+W \quad \text { and } \quad e: W \rightarrow H W+R Y
$$

where $V, W$ are finitely presentable. Then we can form an equation morphism 


$$
\begin{array}{r}
e \square \equiv V+W \stackrel{[f, \mathrm{inr}]}{\longrightarrow} H V+W \stackrel{H V+e}{\longrightarrow} H V+H W+R Y \\
\downarrow \begin{array}{c}
\text { can }+R Y \\
H(V+W)+R Y
\end{array}
\end{array}
$$

and we have

$$
\left(e^{\dagger} \bullet f\right)^{\dagger}=(e \square f)^{\dagger} \cdot \mathrm{inl},
$$

see 3 .

(vi) Finally, every homomorphism $h: A \rightarrow B$ of $H$-algebras between iterative algebras $A$ and $B$ preserves solutions:

$$
h \cdot e^{\dagger}=(h \bullet e)^{\dagger}: X \rightarrow B
$$

for every equation morphism $e: X \rightarrow H X+A$.

Example 4.5. The rational monad of $H_{\lambda}$ is the monad $\mathbb{R}_{\lambda}$ of rational $\lambda$-terms with constants: to every presheaf $Y$ it assigns the presheaf $R_{\lambda}(Y)$ defined precisely as $R_{\lambda}$ in Example 2.2(v) except that in every context $\Gamma$ we can also use elements of $Y(\Gamma)$ to label the leaves.

More detailed: we first define the set $R_{\lambda}^{\prime}(Y)(\Gamma)$ of rational trees in context $\Gamma$ with constants from $Y$. It consists of all rational trees of the form (2.2) such that

a node labelled by an element of $Y(\Gamma)$ is a leaf.

By using the $\alpha$-conversion precisely as in Example 2.2(iii), we obtain the desired presheaf

$$
R_{\lambda}(Y)(\Gamma)=R_{\lambda}^{\prime}(Y)(\Gamma) / \sim_{\alpha}
$$

It is again pointed; the pointing $i^{R_{\lambda}(Y)}: V \rightarrow R_{\lambda}(Y)$ assigns to every variable the corresponding singleton tree. And $R_{\lambda}(Y)$ is canonically an $H_{\lambda}$-algebra. We define

$$
\eta_{Y}: Y \rightarrow R_{\lambda}(Y)
$$

to assign to every element of $Y(\Gamma)$ the corresponding singleton tree. This is the free iterative $H_{\lambda}$-algebra on $Y$, the proof is completely analogous to that of Theorem 2.17.

Definition 4.6. A point-strong monad is a monad $\mathbb{M}=(M, \eta, \mu)$ on $\mathscr{W}$ together with a point-strength

$$
s_{(X, x),(Y, y)}:(M X) \otimes Y \rightarrow M(X \otimes Y)
$$

see Definition 3.3, such that $s$ preserves the unit:

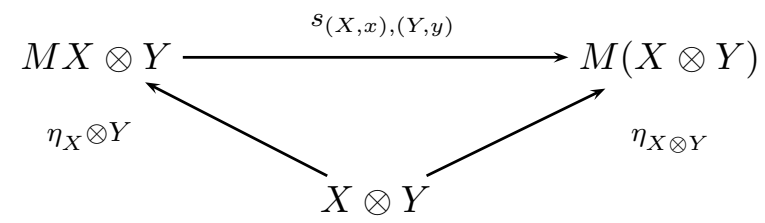

and the multiplication:

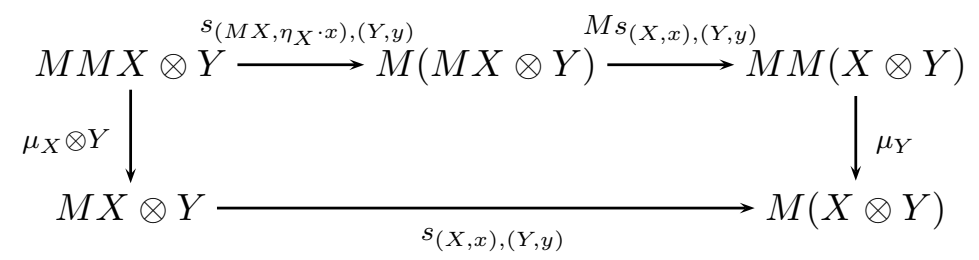


Example 4.7. By our assumption that $H$ be finitary we know that all terminal coalgebras for $H(-)+X$ exist, this follows from [15], see also [5]. Equivalently, all free completely iterative algebras for $H$ exist (cf. Example 2.9), and they yield the object map of a monad $\mathbb{T}=\left(T, \eta^{T}, \mu^{T}\right)$. This monad is the free completely iterative monad on the endofunctor $H$, see [17]. The monad multiplication $\mu_{X}^{T}: T T X \rightarrow T X$ is the unique algebra homomorphism extending $\operatorname{id}_{T X}$, i.e., such that

$$
\mu_{X}^{T} \cdot \tau_{T X}=\tau_{X} \cdot H \mu_{X}^{T} \quad \text { and } \quad \mu_{X}^{T} \cdot \eta_{T X}^{T}=\operatorname{id}_{T X} .
$$

Theorem 4.8. The free completely iterative monad $\mathbb{T}$ of a point-strong endofunctor $H$ is point-strong.

Remark. The strength of $\mathbb{T}$ will be proved to be the unique natural transformation $s^{T}$ for which the diagram

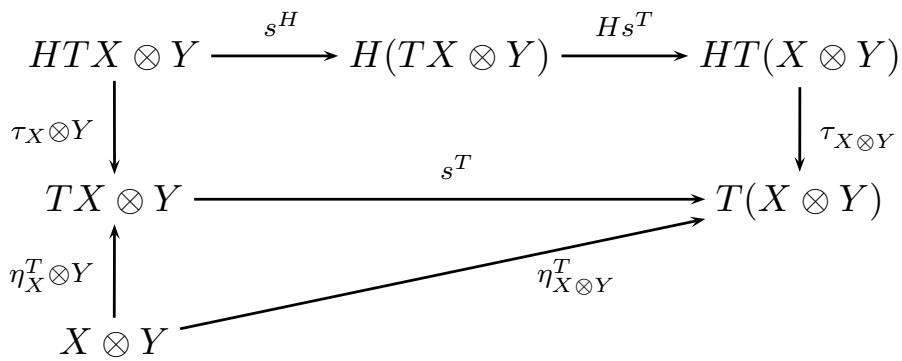

commutes. Note that we have dropped the subscripts indicating the components of the natural transformations $s^{H}$ and $s^{T}$ above; from now on we shall frequently do this when components of natural transformations are clear from the context.

Proof. (a) Let $(X, x)$ and $(Y, y)$ be pointed objects. For every morphism $f: X \otimes Y \rightarrow T Z$ there exists a unique morphism $f^{b}: T X \otimes Y \rightarrow T Z$ such that that the diagram

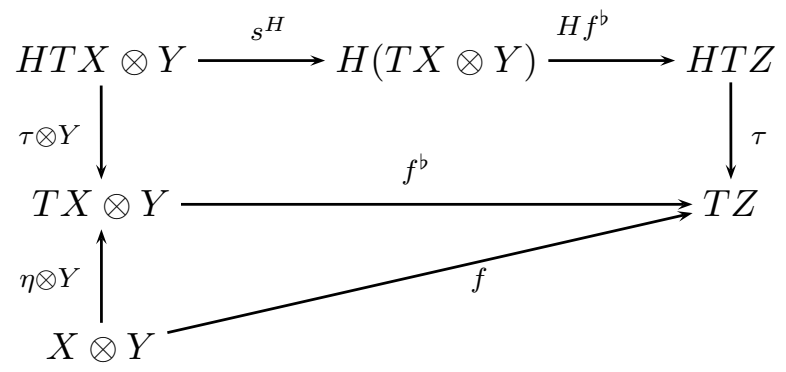

commutes. Indeed, the algebra $T Z$ is completely iterative. Due to $(H T X+Y) \otimes Y=$ $H T X \otimes Y+X \otimes Y$, see Assumption 4.1(3), we obtain an equation morphism in $T Z$ as follows:

$$
T X \otimes Y \stackrel{\left[\tau_{X}, \eta_{X}^{T}\right]^{-1} \otimes Y}{\longrightarrow} H T X \otimes Y+X \otimes Y \stackrel{s_{X, Y}^{H}+f}{\longrightarrow} H(T X \otimes Y)+T Z .
$$

Its unique solution is denoted by $f^{b}$. It is characterized by the commutative diagram 


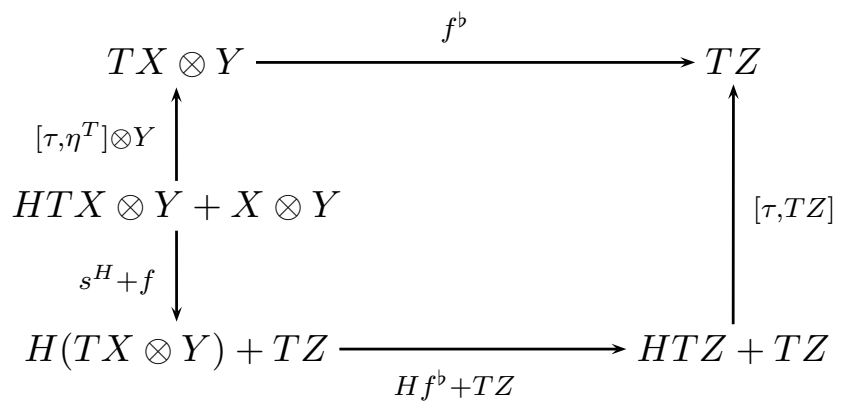

It is easy to verify that this diagram commutes iff (4.11) does.

(b) Put

$$
s_{(X, x),(Y, y)}^{T}=\left(\eta_{X \otimes Y}^{T}\right)^{b}: T X \otimes Y \rightarrow T(X \otimes Y) .
$$

In other words, we define the components of $s^{T}$ via (4.11) uniquely.

(b1) $s^{T}$ is natural: the squares

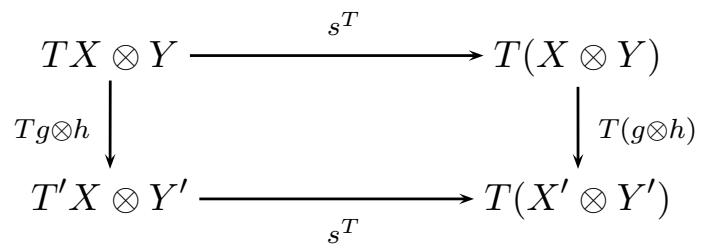

commute for all morphisms $g$ and $h$ of $I / \mathscr{W}$ since both passages form $f^{b}$ for

$$
f=\eta_{X^{\prime} \otimes Y^{\prime}}^{T} \cdot(g \otimes h): X \otimes Y \rightarrow T\left(X^{\prime} \otimes Y^{\prime}\right) .
$$

Indeed, for the upper passage, $f^{b}=T(g \otimes h) \cdot s^{T}$, use the following diagram:

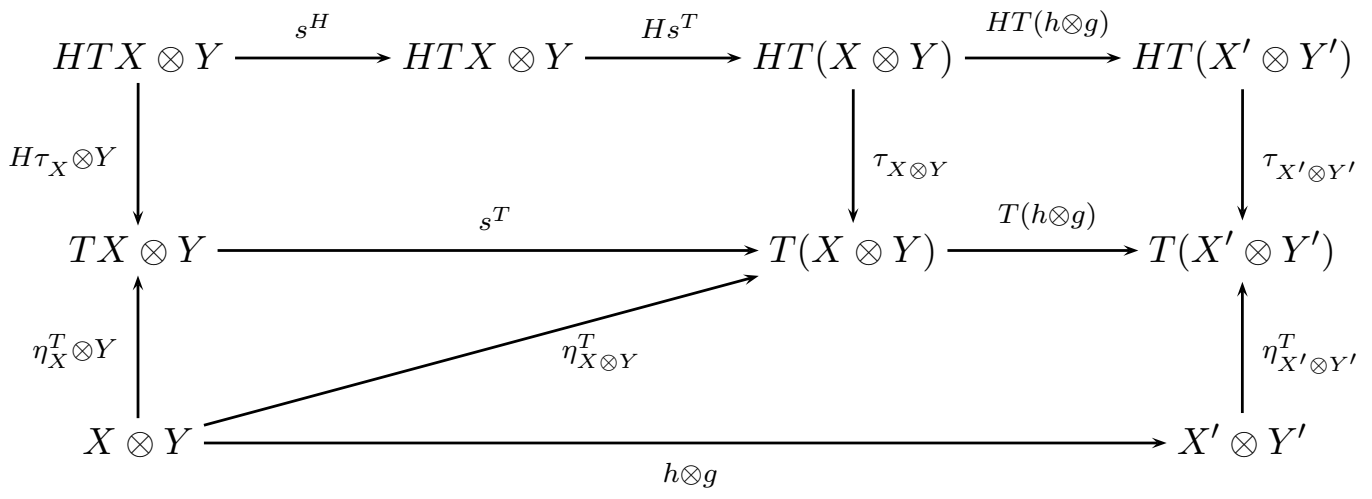

The two left-hand parts form Diagram (4.11), the remaining two commute by naturality of $\tau$ and $\eta$.

The lower passage $f^{b}=s^{T} \cdot(T g \otimes h)$ follows from the following diagram: 


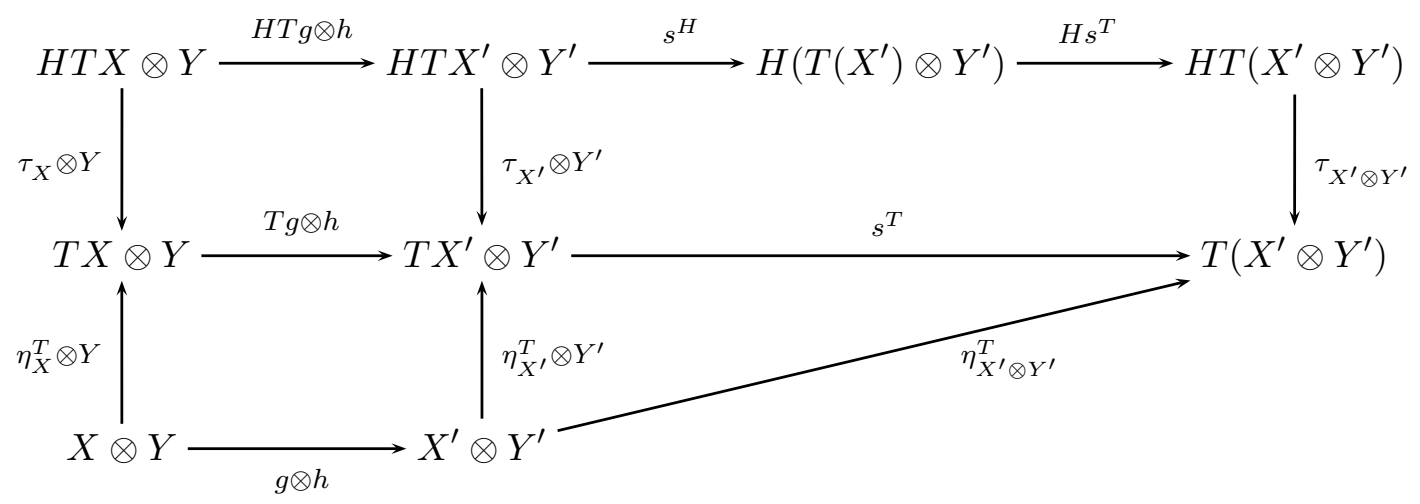

The right-hand parts form Diagram (4.12), the left-hand ones commute by naturality of $\tau$ and $\eta^{T}$.

(b2) $s^{T}$ is a point-strength of the endofunctor $T$. Indeed, the axiom

$$
s_{(X, x)(V, v)}^{T}=\operatorname{id}_{T(X)}
$$

follows from the fact that if $(Y, y)=\left(V\right.$,id), then Diagram (4.10) commutes with $\operatorname{id}_{T(X)}$ in lieu of $s^{T}$. To verify the Axiom (3.3), apply (a) to $f=\eta_{X \otimes Y \otimes Z}^{T}$ : we prove that the lower passage of (3.3) serves as $f^{b}$. In detail, the diagram

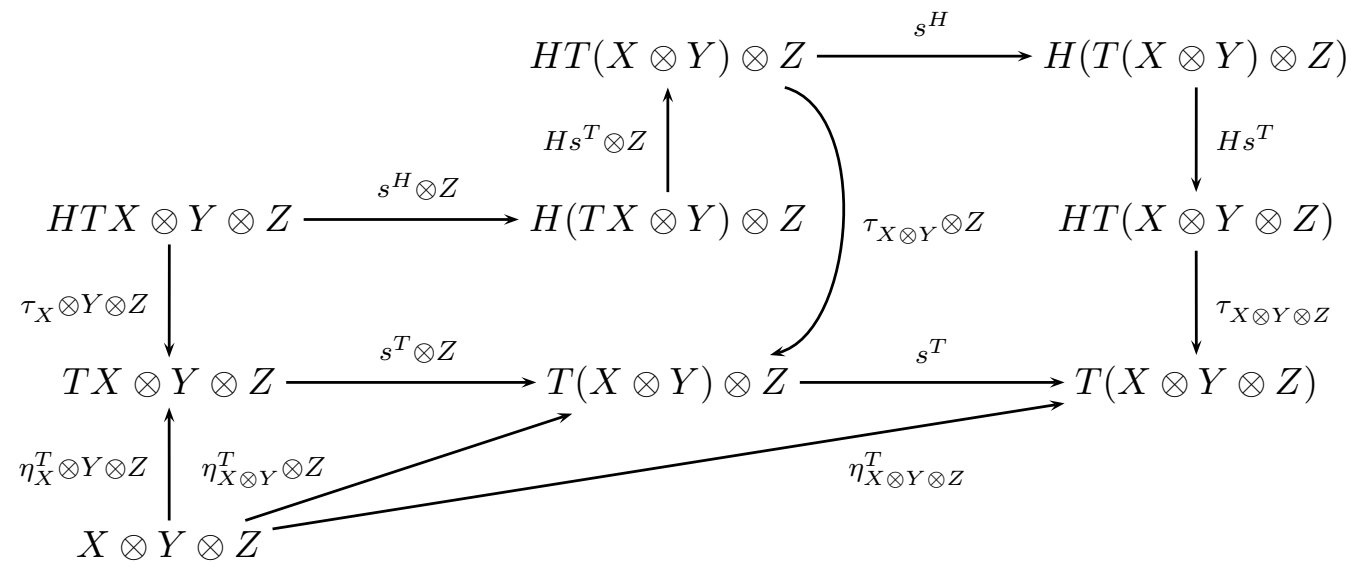

commutes. Indeed, all inner parts commute by two applications of (4.10).

(b3) It remains to verify the axioms of Definition 4.6. For (4.7) use the lower triangle of Diagram (4.10). For (4.8) apply (a) to

$$
f=s^{T}: T X \otimes Y \rightarrow T(X \otimes Y) .
$$

We prove that both passages of (4.8) serve as $f^{b}$. For the lower passage, $\left(s^{T}\right)^{b}=s^{T} \cdot \mu^{T} \otimes Y$, use the following diagram 


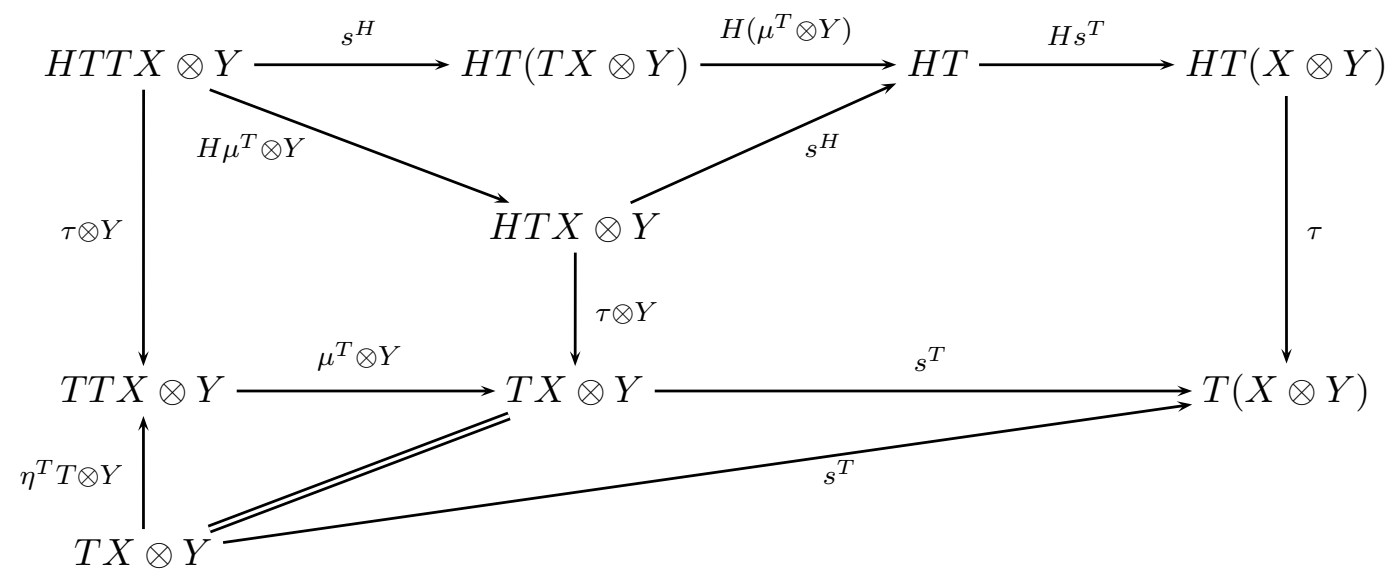

The upper left-hand part is Equation (4.9), the lower one commutes by the monad axiom $\mu^{T} \cdot \eta^{T} T=\mathrm{id}$, the upper triangle is the naturality of $s^{H}$, and the right-hand part follows from (4.10).

For the upper passage, $\left(s^{T}\right)^{b}=\mu^{T} \cdot T s^{T} \cdot s^{T}$, use the following diagram

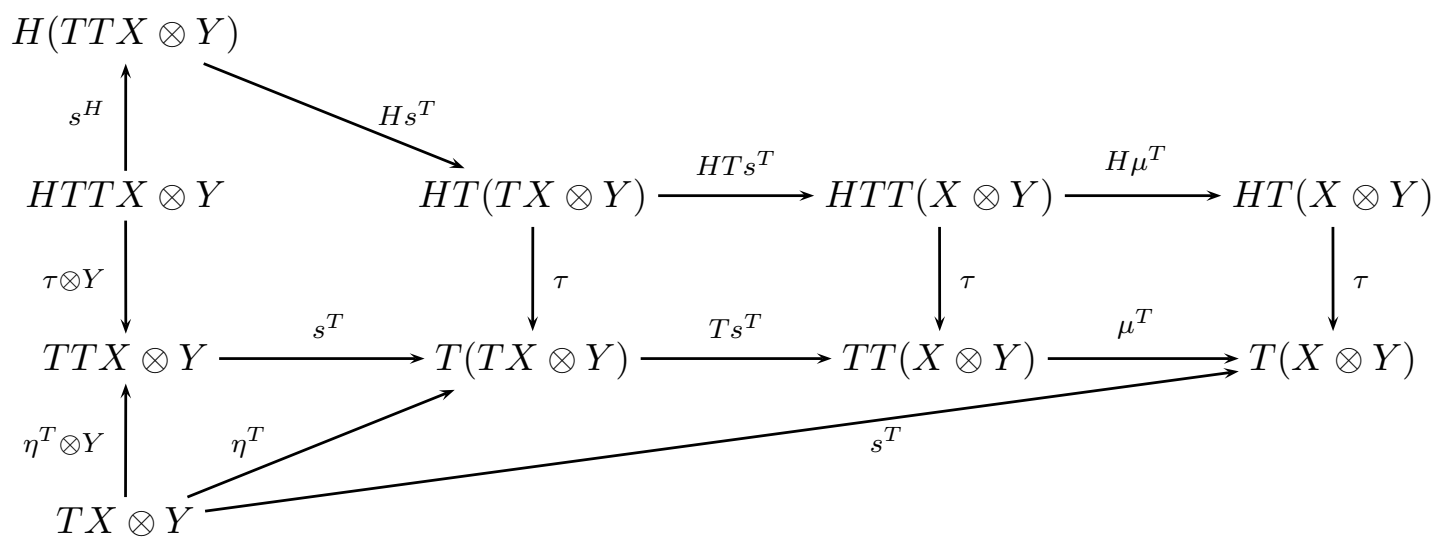

The three upper squares commute due to (4.10), the naturality of $\tau$ and (4.9). The lower triangles commute due to (4.10), the naturality of $s^{T}$ and $\mu^{T} \cdot \eta^{T} T=\mathrm{id}$.

Remark 4.9. Recall from Example 2.9 that $T=H T+$ Id with injections $\tau$ and $\eta^{T}$. From the Diagram (4.10) we see that the strength $s^{T}$ then has the form

$$
s^{T}=H s^{T} \cdot s^{H}+X \otimes Y: H T X \otimes Y+X \otimes Y \rightarrow H T(X \otimes Y)+X \otimes Y .
$$

Theorem 4.10. The rational monad of a point-strong endofunctor is point-strong.

Remark. The strength of $\mathbb{R}$ will be proved to be the unique natural transformation $s^{R}$ for which the diagram 


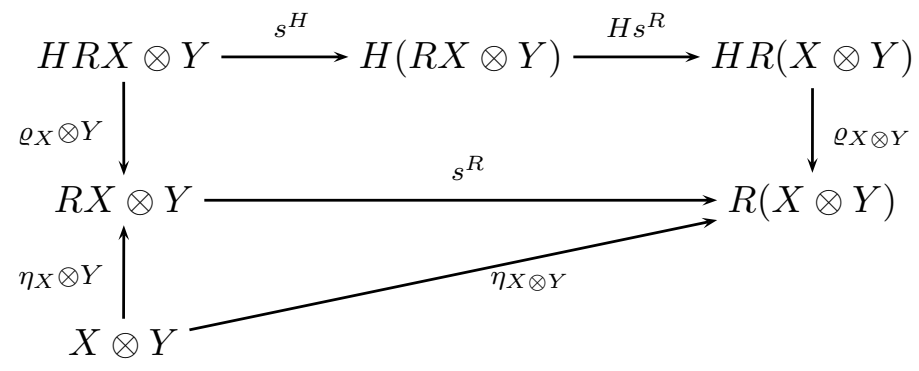

commutes.

Proof. (a) Given pointed objects $(X, x)$ and $(Y, y)$, we prove that for every morphism $f: X \otimes$ $Y \rightarrow R Z$ there exists a unique morphism $f^{b}: R X \otimes Y \rightarrow R Z$ such that the following diagram commutes:

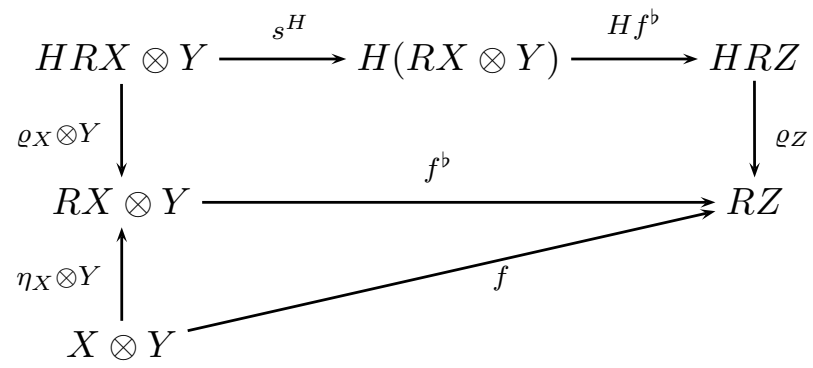

(a1) Assume that $Y$ is finitely presentable. Recall $R Y=$ colim $\mathrm{Eq}_{Y}$ from Remark 4.4. For every object

$$
e: W \rightarrow H W+X \quad \text { in } \mathrm{EQ}_{X}
$$

define, using the distributivity $(H W+Y) \otimes Y=H W \otimes Y+X \otimes Y$ (see Assumption 4.1(3)), the equation morphism

$$
\hat{e} \equiv W \otimes Y \stackrel{e \otimes Y}{\longrightarrow} H W \otimes Y+X \otimes Y \stackrel{s^{H}+f}{\longrightarrow} H(W \otimes Y)+R Z .
$$

Since $W \otimes Y$ is finitely presentable by Assumption 4.1(2), we obtain the unique solution $\hat{e}^{\dagger}: W \otimes Y \rightarrow R Z$, and those solutions form a cocone of the diagram $\mathrm{Eq}_{X} \otimes Y$. Indeed, given a connecting morphism

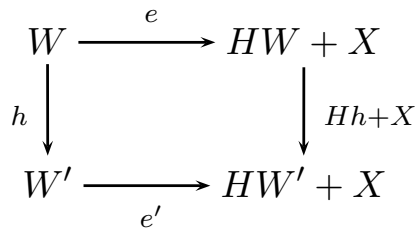

then $h \otimes Y$ is a coalgebra homomorphism from $\hat{e}$ to $\hat{e}^{\prime}$ :

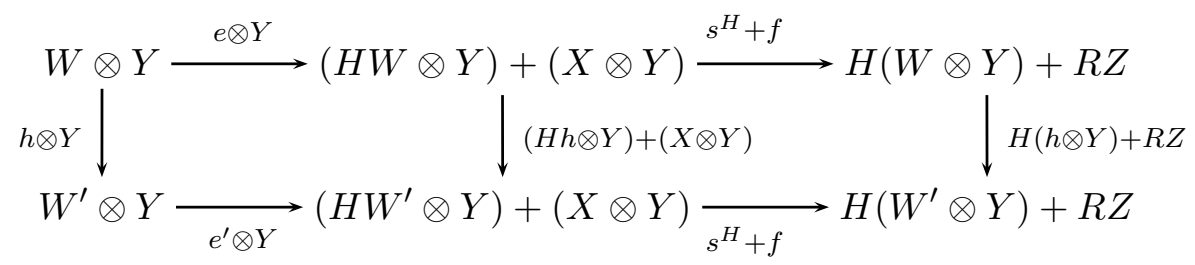


which implies, by Remark 4.4(v) that

$$
\hat{e}^{\dagger}={\widehat{e^{\prime}}}^{\dagger} \cdot(h \otimes Y)
$$

Consequently, we can define

$$
f^{b}: R X \otimes Y \rightarrow R Z
$$

by the commutativity of the triangles

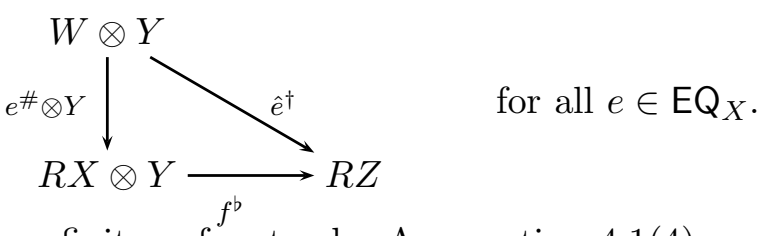

Indeed, since $-\otimes Y$ is a finitary functor by Assumption 4.1(4), we see that $R X \otimes Y$ is a colimit of $\mathrm{Eq}_{Y} \otimes Y$ with the colimit cocone $e^{\#} \otimes Y$. We now verify that the Diagram (4.14) commutes. Consider the diagram below:

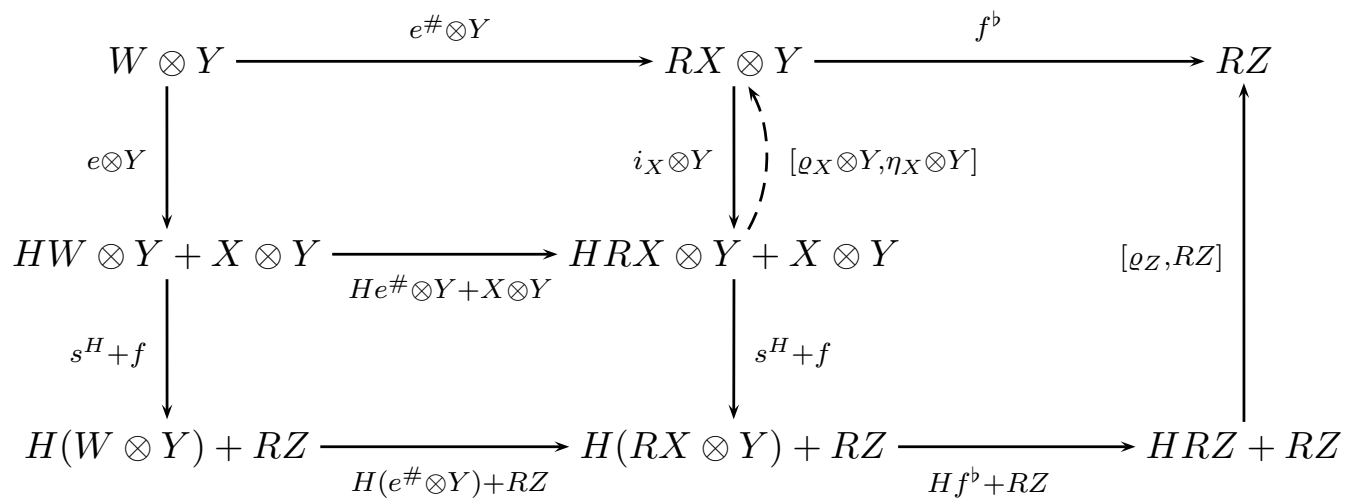

Notice first that the left-hand edge is $\hat{e}$. The upper left-hand part commutes by (4.4), and the lower one does by naturality of $s^{H}$. The outside of the diagram commutes since $f^{b} \cdot\left(e^{\#} \otimes Y\right)$ is the unique solution of $\hat{e}$ in the iterative algebra $R Z$. Thus, the right-hand part commutes when precomposed by any $e^{\#} \otimes Y$. So since the latter morphisms are collectively epimorphic (being the injections of colim $\mathrm{Eq}_{X} \otimes Y$ ), we see that the right-hand part commutes. Now we use that $i_{X}$ is an isomorphism with the inverse $\left[\varrho_{X}, \eta_{X}\right]$, see Equation (4.5), which implies

$$
\left[\varrho_{X} \otimes Y, \eta_{X} \otimes Y\right]=\left(i_{X} \otimes Y\right)^{-1} .
$$

Finally observe that the two coproduct components of the right-hand part of (4.17) yield precisely the upper and lower parts of (4.14) - this proves that (4.14) commutes.

It only remains to prove the uniqueness of $f^{b}$. So suppose we have some $f^{b}$ such that Diagram (4.14) commutes. Equivalently, the right-hand part of (4.17) commutes, and this implies that $f^{b} \cdot\left(e^{\#} \otimes Y\right)$ is, for every $e$ in $\mathrm{EQ}_{X}$, a solution of $\hat{e}$. This determines $f^{b}$ uniquely.

(a2) Let $Y$ be arbitrary. Then since $\mathscr{W}$ is locally finitely presentable we can express $Y$ as a filtered colimit

$$
Y=\operatorname{colim}_{q \in Q} Y^{q} \quad \text { with colimit cocone } y^{q}: Y^{q} \rightarrow Y
$$


of finitely presentable objects $Y^{q}$. By Assumption 4.1(2) the unit object $I$ is finitely presentable, thus the given pointing of $Y$ :

$$
y: I \rightarrow \operatorname{colim}_{q \in Q} Y^{q}
$$

factorizes through some $y^{q}$. The diagram above being filtered, we can assume that this factorization takes place for every $q \in Q$, in other words, that we have a filtered diagram of pointed objects $Y^{q}$ with colimit $Y$ (and with all the connecting morphisms $Y^{q} \rightarrow Y^{q^{\prime}}$ preserving the pointing).

Given $f: X \otimes Y \rightarrow R Z$, for every $q \in Q$ we know from the previous part (a1) that there exists a unique

$$
f_{q}^{b}: R X \otimes Y^{q} \rightarrow R Z
$$

such that Diagram (4.14) commutes when $f^{b}$ is replaced by $f_{q}^{b}$ and $f$ by

$$
f_{q} \equiv X \otimes Y^{q} \stackrel{X \otimes y^{q}}{\longrightarrow} X \otimes Y \stackrel{f}{\longrightarrow} R Z .
$$

This defines a unique $f^{b}: R X \otimes Y \rightarrow R Z$ with

$$
f_{q}^{b}=f^{b} \cdot\left(R X \otimes y^{q}\right) \quad \text { for all } q \in Q .
$$

Now Diagram (4.14) commutes because $H R X \otimes Y=\operatorname{colim}_{q \in Q} H R X \otimes Y^{q}$ as well as $X \otimes Y=$ $\operatorname{colim}_{q \in Q} X \otimes Y^{q}$. And $f^{b}$ is uniquely determined by this commutativity; indeed, for any $f^{b}$ such that (4.14) commutes one easily verifies that (4.18) holds using the uniqueness of $f_{q}^{b}$ from part (a1).

(b) Analogously to the proof of Theorem 4.8 put

$$
s_{(X, x),(Y, y)}^{R}=\eta_{X \otimes Y}^{b}: R X \otimes Y \rightarrow R(X \otimes Y) .
$$

The verification that $s^{R}$ is the desired strength is analogous to the above proof: just replace $T$ by $R$ (and $\tau$ by $\varrho$ ).

Remark 4.11. The proofs of Theorems 4.8 and 4.10 have the same structure, and also the proof that the monad $\mathbb{F}_{\lambda, \Sigma}$ is point-strong can proceed analogously:

Let $H$ be a point-strong endofunctor of $\mathscr{W}$ and let $(\hat{M}, \hat{\mu}, \hat{\eta})$ be a monad. Suppose that a natural transformation $\alpha: H \hat{M} \rightarrow \hat{M}$ has the property that for every morphism $f: X \otimes Y \rightarrow M Z$ there exists a unique morphism $f^{b}: M X \otimes Y \rightarrow M Z$ with $f=f^{b} \cdot\left(\hat{\eta}_{X} \otimes Y\right)$ and $f^{b} \cdot\left(\alpha_{X} \otimes Y\right)=\alpha_{X} \cdot H f^{b} \cdot s^{H}$. Then $M$ is a point-strong $\operatorname{monad}$ w.r.t. $s^{M}=\hat{\eta}_{X \otimes Y}^{b}$.

Remark 4.12. The morphisms

$$
\varrho_{X}: H R X \rightarrow R X \quad \text { and } \quad \eta_{X}: X \rightarrow R X
$$

of (4.13) are coproduct injections of

$$
R X=H R X+X
$$

as proved in 2]. From diagram (4.13) we conclude that the strength of $\mathbb{R}$,

$$
s^{R}: R X \otimes Y \rightarrow R(X \otimes Y)
$$

whose domain is $H R X \otimes Y+X \otimes Y$ by 4.1 (2) and codomain is $H R(X \otimes Y)+X \otimes Y$, has the form

$$
s^{R}=H s^{R} \cdot s^{H}+X \otimes Y .
$$


Corollary 4.13. For a point-strong endofunctor $H$ the free iterative $H$-algebra

$$
R I
$$

on the unit object is an $H$-monoid w.r.t. the unit $i=\eta_{I}: I \rightarrow R I$ and the multiplication

$$
m \equiv R I \otimes R I \stackrel{s_{I, R I}^{R}}{\longrightarrow} R R I \stackrel{\mu_{I}}{\longrightarrow} R I .
$$

Proof. Indeed, the unit laws are obvious:

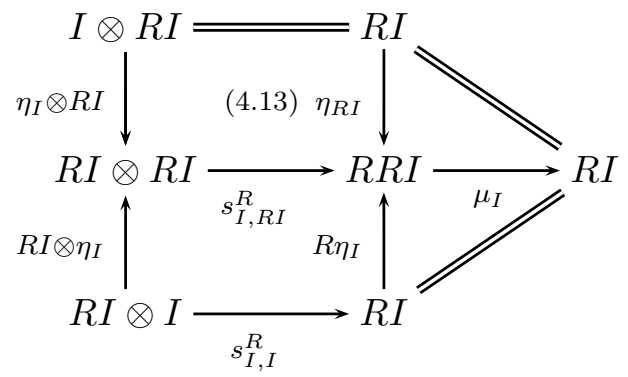

where the lower square commutes by the naturality of $s^{R}$. For the associativity we have the following commutative diagram

$R I \otimes m$

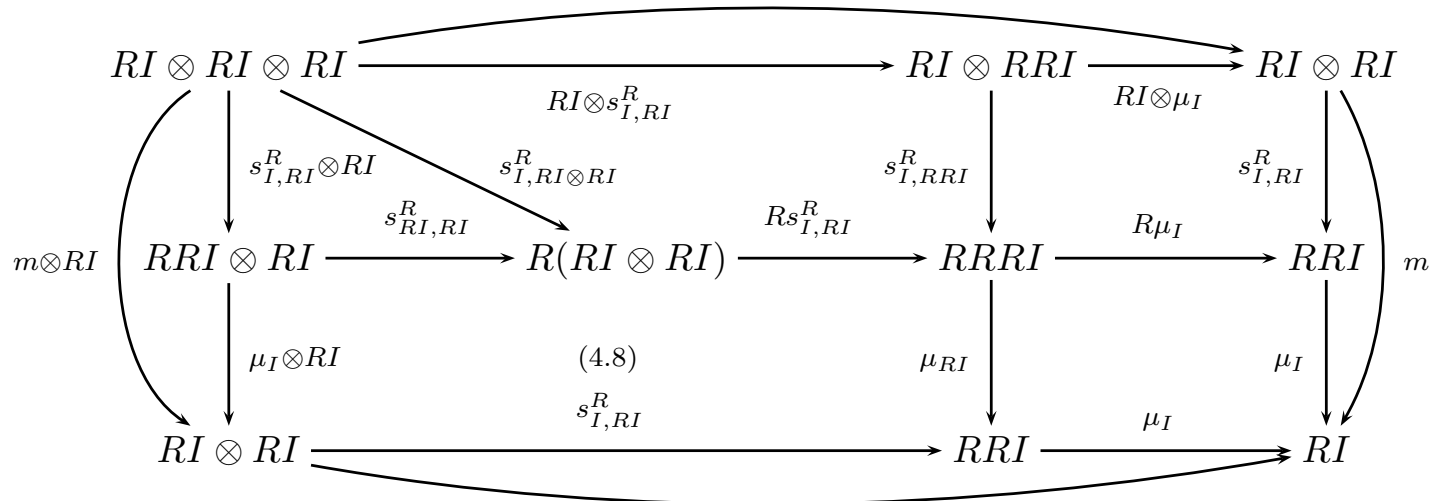

$m$

Finally, the Diagram (3.4) commutes due to (4.13):

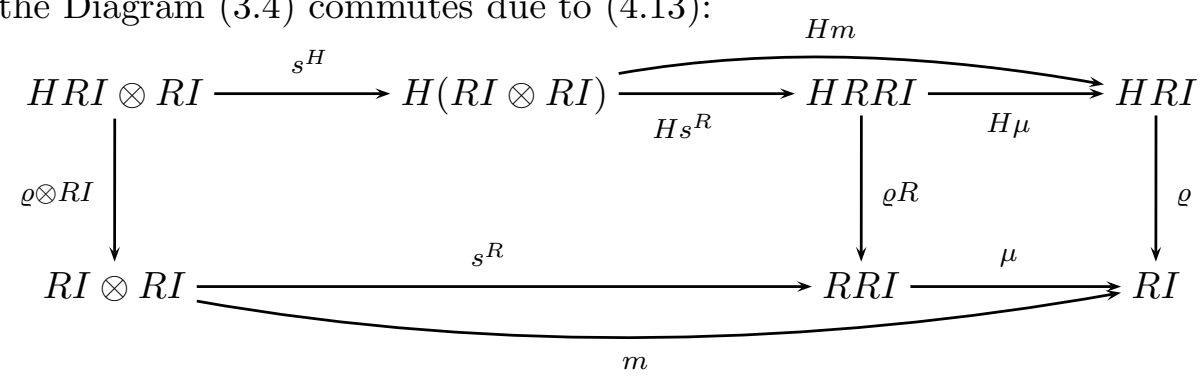

Corollary 4.14. The free completely iterative $H$-algebra

TI

on the unit object is an $H$-monoid w.r.t. $\eta_{I}^{T}$ and $\mu_{I}^{T} \cdot s_{I, T I}^{T}$.

The proof is completely analogous to the previous one. 
Notation 4.15. Let

$$
e: W \rightarrow H W+I
$$

be a flat equation morphism in $I$, and let a pointing of $W$ be given.

(i) $e^{\#}: W \rightarrow R I$ denotes the colimit morphism of $R I=\operatorname{colim} \mathrm{Eq}_{I}$ and

$$
\widehat{e^{\#}}: R W \rightarrow R I
$$

its unique extension to a homomorphism of $H$-algebras.

(ii) $e * W$ denotes the following equation morphism in $W$ :

$$
e * W \equiv W \otimes W \stackrel{e \otimes W}{\longrightarrow} H W \otimes W+W \stackrel{s^{H}+W}{\longrightarrow} H(W \otimes W)+W .
$$

(iii) $\langle e\rangle: W \otimes W+W \rightarrow H(W \otimes W+W)+I$ denotes the flat equation morphism whose left-hand component is

$$
\begin{aligned}
\langle e\rangle \cdot \mathrm{inl} \equiv W \otimes W \stackrel{e * W}{\longrightarrow} & H(W \otimes W)+W \\
& H(W \otimes W)+e \mid \\
& H(W \otimes W)+H W+I \stackrel{\text { can }+I}{\longrightarrow} H(W \otimes W+W)+I
\end{aligned}
$$

and the right-hand one is

$$
\langle e\rangle \cdot \mathrm{inr} \equiv W \stackrel{e}{\longrightarrow} H W+I \stackrel{H \mathrm{inr}+I}{\longrightarrow} H(W \otimes W+W)+I .
$$

Lemma 4.16. For every flat equation morphism $e: W \rightarrow H W+I$ with $W$ pointed the square

commutes.

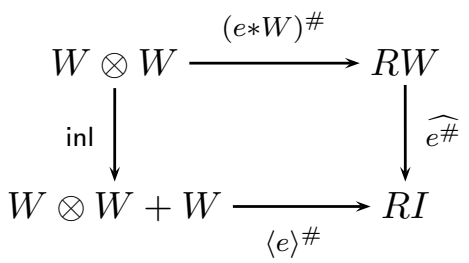

Proof. Notice that $\langle e\rangle$ is precisely of the form $e \square f$ from Remark 4.4(vi) for $f=e * W$. Also recall from Remark 4.4(iv) that $\langle e\rangle^{\#}=(\eta \bullet\langle e\rangle)^{\dagger}$ and similarly $(e * W)^{\#}=(\eta \bullet(e *$ $W))^{\dagger}$. We shall also use that the $H$-algebra homomorphism $\widehat{e^{\#}}$ preserves solutions (cf. Remark 4.4(vii)). Thus, we compute

$$
\begin{aligned}
& \widehat{e^{\#}} \cdot(e * W)^{\#}=\widehat{e^{\#}} \cdot(\eta \cdot(e * W))^{\dagger} \\
& =\left(\left(\widehat{e^{\#}} \cdot \eta\right) \bullet(e * W)\right)^{\dagger} \quad 4.4 \text { (vii) } \\
& =\left(e^{\#} \bullet(e * W)\right)^{\dagger} \quad \widehat{e^{\#}} \text { extends } e^{\#} \\
& =\left((\eta \cdot e)^{\dagger} \bullet(e * W)\right)^{\dagger} \quad \text { 4.4(iv) } \\
& =(\eta \cdot e \square e * W)^{\dagger} \cdot \mathrm{inl} \\
& =(\eta \bullet(e \square(e * W)))^{\dagger} \cdot \text { inl } \quad \text { obvious } \\
& =(e \square(e * W))^{\#} \cdot \text { inl } 4.4(\text { iv }) \\
& =\langle e\rangle^{\#} \cdot \mathrm{inl} \quad \text { 4.4. (vi) }
\end{aligned}
$$


This completes the proof.

Theorem 4.17. Let $H$ be a finitary, point-strong endofunctor. Then the $H$-monoid $R I$ above is the initial iterative $H$-monoid.

That is, for every $H$-monoid $A$ there exists precisely one morphism $h: R I \rightarrow A$ which is both a monoid homomorphism and a homomorphism of $H$-algebras.

Proof. (1) Given an iterative $H$-monoid

$$
\bar{a}: H \bar{A} \rightarrow \bar{A}, \quad \bar{i}: I \rightarrow \bar{A} \quad \text { and } \quad \bar{m}: \bar{A} \otimes \bar{A} \rightarrow \bar{A}
$$

we know that there is a unique $H$-algebra homomorphism

$$
h: R I \rightarrow \bar{A} \quad \text { with } \quad h \cdot \eta_{I}=\bar{i} .
$$

It is our task to prove that $h$ preserves multiplication:

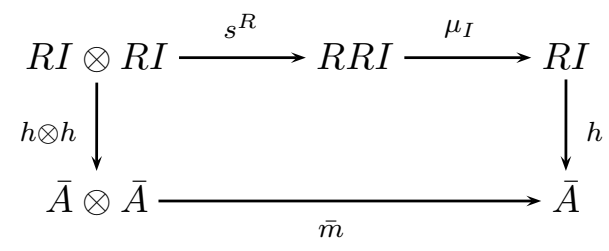

(2) Recall $R I=$ colim $\mathrm{Eq}_{I}$ from Remark 4.4. We can substitute $\mathrm{EQ}_{I}$ by the category of all $e: W \rightarrow H W+I$ with $W$ pointed. The argument is as in (a2) of Theorem 4.10, We indicate pointing by writing $W_{\bullet}$ instead of $W$ (this stands for the notation $\left(W, i^{W}\right)$ ). We know that $\otimes$ is finitary, thus

$$
R I \otimes R I=\operatorname{colim} \mathrm{Eq}_{I} \otimes \mathrm{Eq}_{I}
$$

with the colimit cocone

$$
e^{\#} \otimes e^{\#}: W_{\bullet} \otimes W_{\bullet} \rightarrow R I \otimes R I .
$$

It is thus sufficient to prove that for every $e$ the square

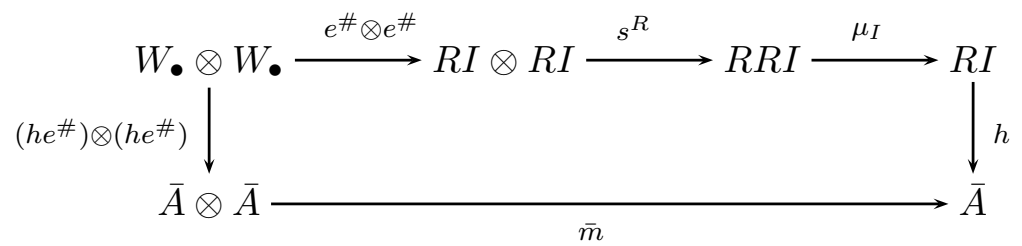

commutes.

For every flat equation morphism $e: W \rightarrow H W+I$ recall $\langle e\rangle$ from Notation 4.15(iii) and put

$$
\bar{e} \equiv W \stackrel{e}{\longrightarrow} H W+I \stackrel{H W+\bar{i}}{\longrightarrow} H W+\bar{A} .
$$

We prove that (4.23) commutes by verifying that the two sides of the square are both the left-hand part of the solution $\bar{f}^{\dagger}: W \otimes W+W \rightarrow \bar{A}$ of the equation morphism $\bar{f}$ for

$$
f=\langle e\rangle: W \otimes W+W \rightarrow H(W \otimes W+W)+I .
$$

(3) Proof of the upper passage of (4.23):

$$
h \cdot \mu_{I} \cdot s^{R} \cdot\left(e^{\#} \otimes e^{\#}\right)=\bar{f}^{\dagger} \cdot \operatorname{inl}: W \otimes W \rightarrow \bar{A} .
$$


Since $h: R I \rightarrow \bar{A}$ preserves solutions by Remark 4.4(vii), and since $f^{\#}$ is a solution of $\eta_{I} \bullet f$ as mentioned in Remark 4.4(iv), the composite $h \cdot f^{\#}$ is a solution of the equation morphism $\bar{f}$ : indeed, $h$ takes $\eta_{I} \bullet f$ to $\bar{f}$ due to the diagram

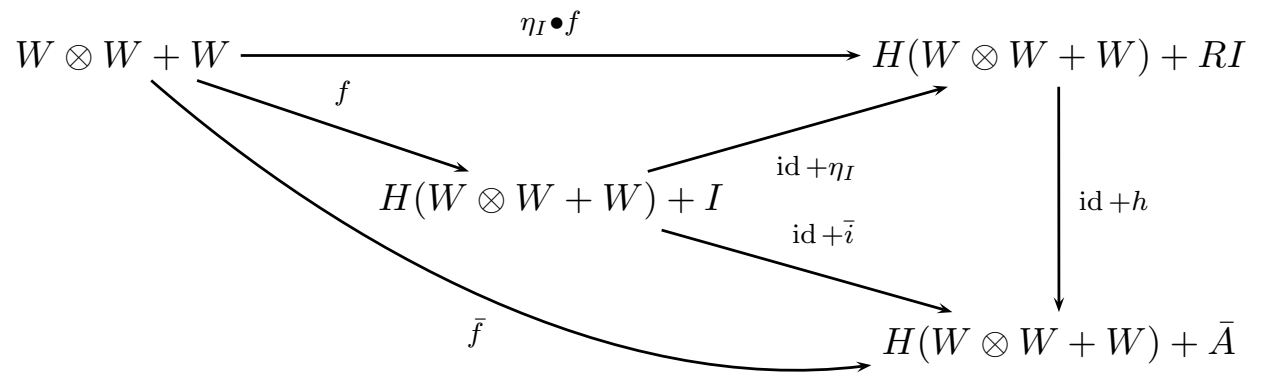

Shortly, the triangle

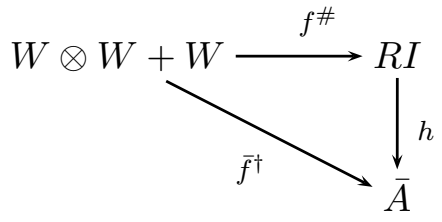

commutes.

Observe that also the triangle

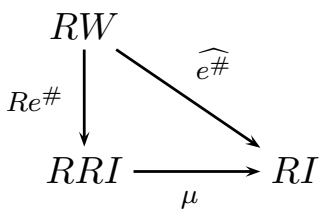

commutes since $R W$ is the free iterative algebra on $\eta_{W}: W \rightarrow R W$ and the three morphisms above are homomorphisms of $H$-algebras which are merged by $\eta_{W}$-indeed:

$$
\widehat{e^{\#}} \cdot \eta_{W}=e^{\#}
$$

as well as

$$
\mu \cdot R e^{\#} \cdot \eta_{W}=\mu \cdot \eta_{R I} \cdot e^{\#}=e^{\#}
$$

Let us verify that

$$
s_{I, W} \cdot\left(e^{\#} \otimes W\right)=(e * W)^{\#} .
$$

Indeed, for $e: W \rightarrow H W+I$ and $f=\eta_{W}$ form $\hat{e}$ as in (4.15) and observe that $\hat{e}=\eta_{W} \bullet(e * W)$ holds. Now recall from Equation (4.19) that $s_{I, W}^{R}=\eta_{W}^{b}$. Thus, we have

$$
\begin{array}{rlrl}
s_{I, W}^{R} \cdot\left(e^{\#} \otimes W\right) & =\eta_{W}^{b} \cdot\left(e^{\#} \otimes W\right) & \\
& =\hat{e}^{\dagger} & & \text { by (4.16) } \\
& =\left(\eta_{W} \bullet(e * W)\right)^{\dagger} & & \\
& =(e * W)^{\#} & & \text { by Remark 4.4(iv). }
\end{array}
$$

We are now in the position to demonstrate (4.25): 


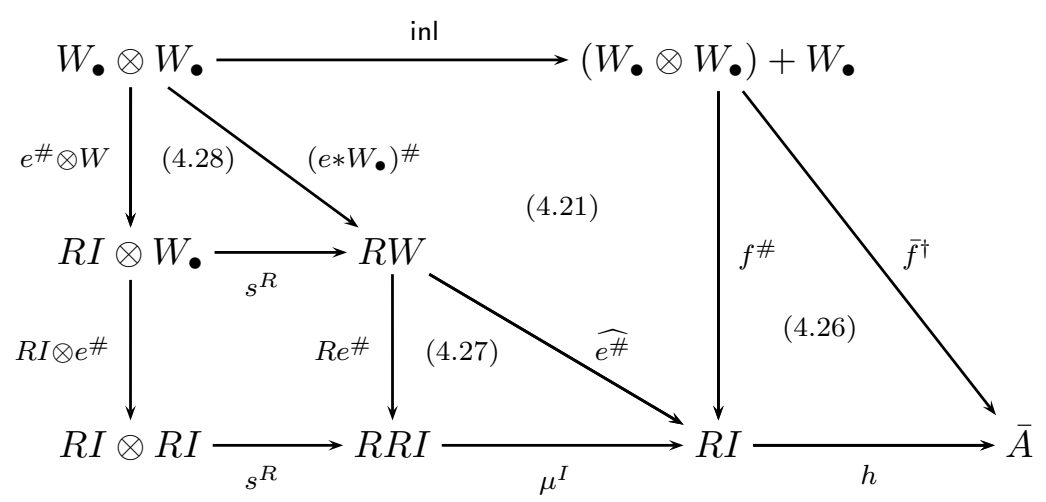

(4) We shall prove for the lower passage of (4.23) that

$$
\bar{m} \cdot\left(\left(h \cdot \bar{e}^{\#}\right) \otimes\left(h \cdot \bar{e}^{\#}\right)\right)=\bar{f}^{\dagger} \cdot \text { inl }: W \otimes W \rightarrow \bar{A} .
$$

Since the homomorphism $h: R I \rightarrow A$ preserves solutions and, by Remark 4.4(iv), $e^{\#}$ is the solution of $\eta_{I} \bullet e$ in $R I$, it follows that $\bar{e}^{\dagger}=h \cdot e^{\#}$ (cf. (4.26)). So we will prove that

$$
\bar{f}^{\dagger}=\left[\bar{m} \cdot\left(\bar{e}^{\dagger} \otimes \bar{e}^{\dagger}\right), \bar{e}^{\dagger}\right]: W \otimes W+W \rightarrow \bar{A} .
$$

To see this it suffices to verify that the following diagram

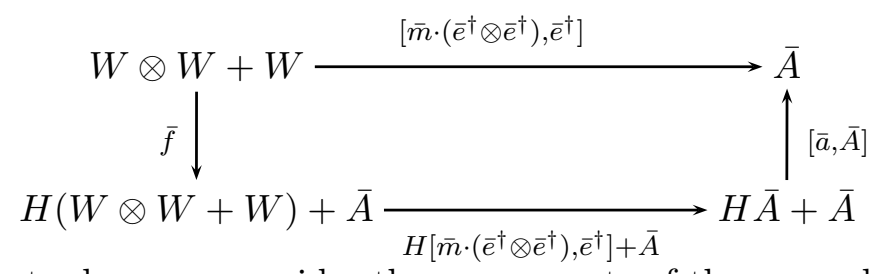

commutes. In order to do so we consider the components of the upper left-hand coproduct separately. For the right-hand component with domain $W$ we obtain

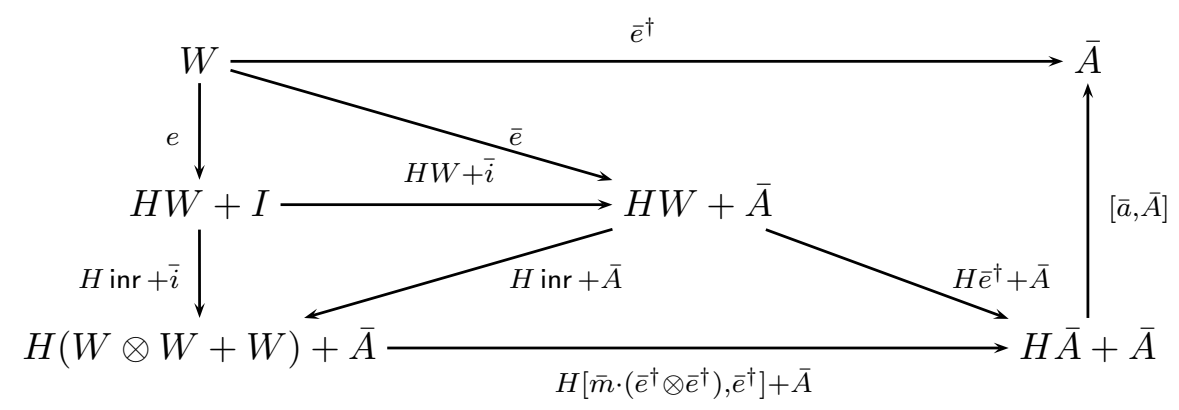

This diagram commutes: the upper right-hand part commutes since $\bar{e}^{\dagger}$ is a solution of $\bar{e}$, and all other inner parts are obvious.

For the left-hand component of (4.29) we prove that the following diagram 


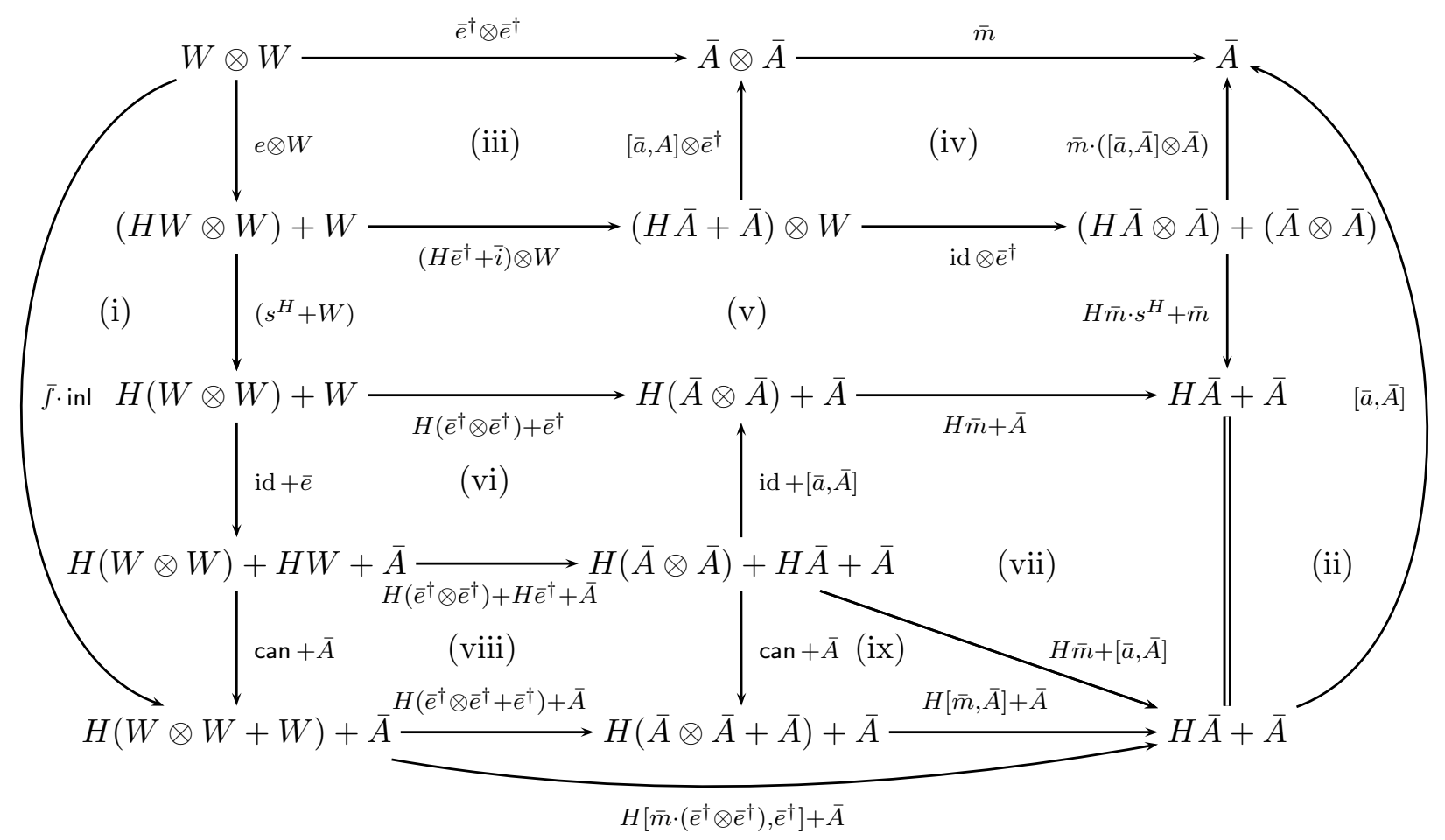

commutes: the left-hand part (i) commutes by the definition of $\bar{f}=\overline{\langle e\rangle}$ and right-hand part (ii) is obvious since $\bar{A}$ is an $H$-monoid, i.e., $\bar{a} \cdot H \bar{m} \cdot s^{H}=\bar{m} \cdot(\bar{a} \otimes \bar{A})$. For part (iii) observe that $(H W \otimes W)+W=(H W+I) \otimes W$ and use Diagram (2.3) and Equation (4.24). In part (iv) we use the distributivity for the object in the lower right-hand corner: $(H \bar{A}+$ $\bar{A}) \otimes \bar{A}=H \bar{A} \otimes \bar{A}+\bar{A} \otimes \bar{A}$ - the commutativity is then obvious. We postpone part (v) to the end. Part (vi) commutes by using (2.3). Parts (vii) and (viii) are trivial. We do not claim that part (ix) commutes, but it clearly does when post-composed with $[\bar{a}, \bar{A}]$, which suffices for the commutativity of the outside of the diagram. Finally, it remains to prove that part (v) commutes: we consider the components of the coproduct $(H W \otimes W)+W$ separately. For the right-hand component we obtain the commutative diagram

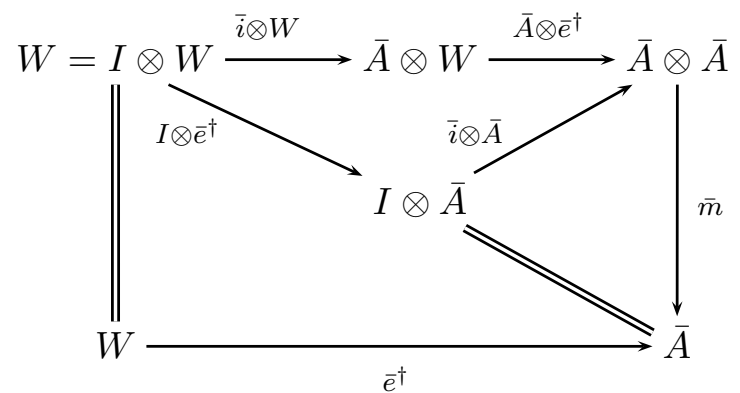

and for the left-hand one consider the diagram below

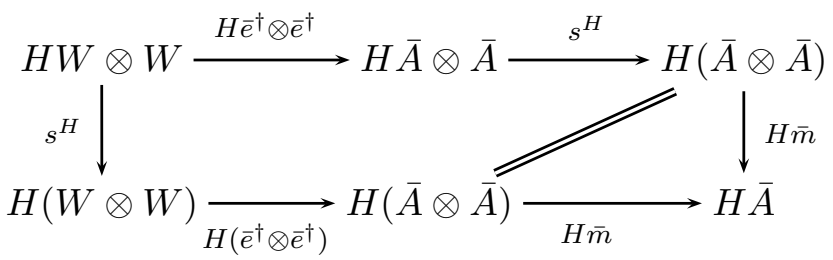


This completes the proof.

Theorem 4.18. Let $H$ be a finitary, point-strong endofunctor. Then the monoid TI from Corollary 4.14 is the initial completely iterative $H$-monoid.

Notice that our proof below uses just the existence of the completely iterative algebras for $H$ (cf. Example 4.7) and not finitariness of $H$ directly.

Proof. Analogously to the preceding proof, for a completely iterative $H$-monoid $(\bar{A}, \bar{i}, \bar{m}, \bar{a})$ we know that there exists a unique $H$-algebra homomorphism

$$
h: T I \rightarrow \bar{A} \quad \text { with } \quad h \cdot \eta_{I}^{T}=\bar{i}
$$

and it is our task to prove that it preserves multiplication:

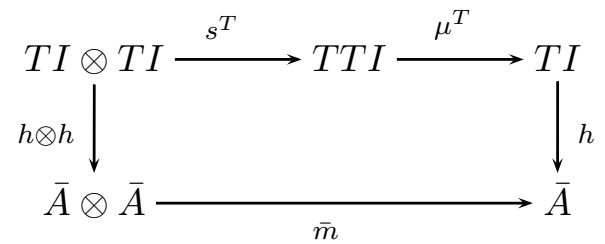

To prove this, we define an equation morphism in $\bar{A}$ :

$$
e: T I \otimes T I=H T I \otimes T I+T I \rightarrow H(T I \otimes T I)+\bar{A}
$$

such that both passages of (4.32) are solutions of $e$ in $\bar{A}$ : put

$$
e=s_{T I, T I}^{H}+h \text {. }
$$

For the upper passage of (4.32) we need to verify that the square

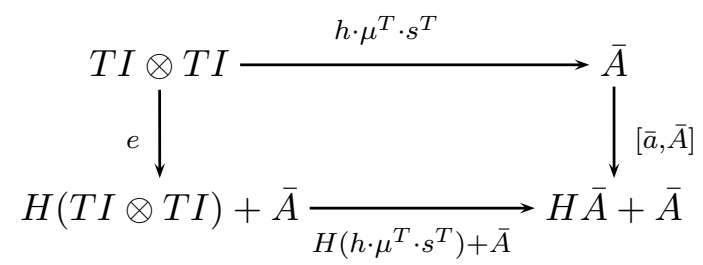

commutes. Consider the components of $H T I \otimes T I+I \otimes T I$ separately. The left-hand component yields

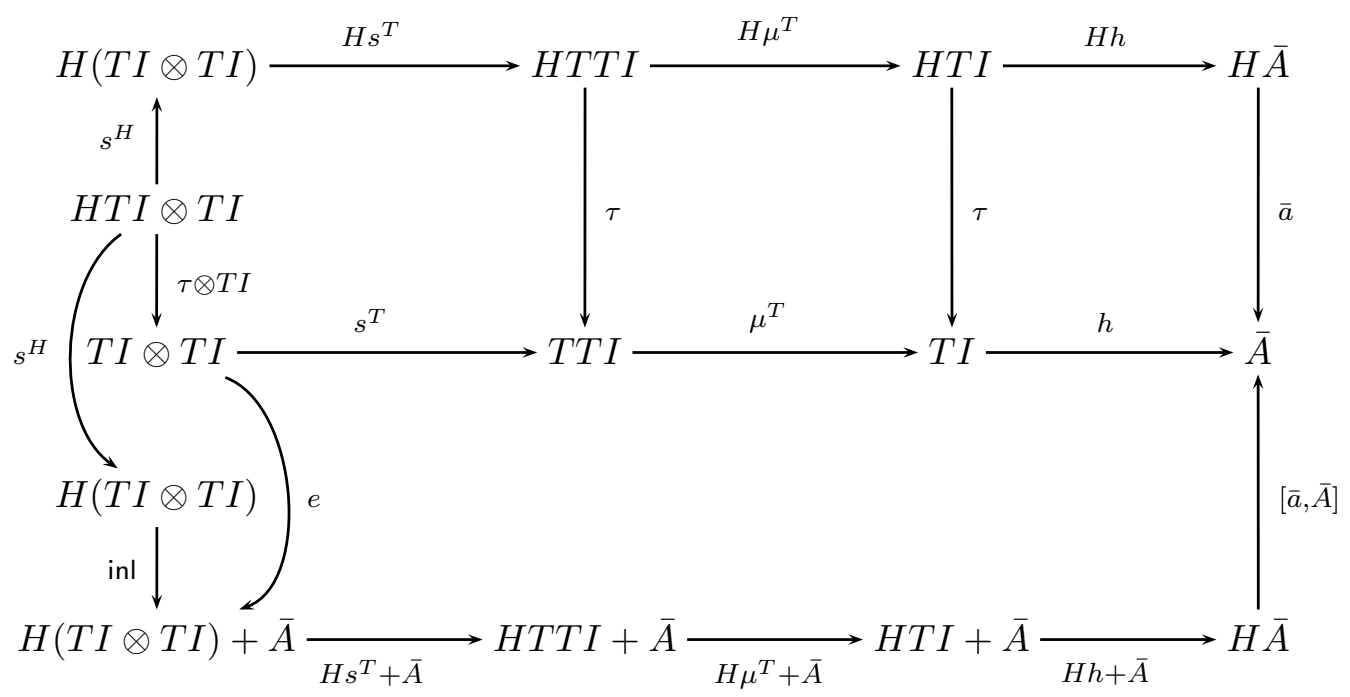


The two upper right-hand squares commute since $\mu^{T}$ and $h$ are homomorphisms of algebras, the upper left-hand square is Diagram (4.10) and the lower left-hand part is obvious. Thus, since the outside of the diagram clearly commutes we see that its lower part (4.33) commutes when extended by the left-hand injection $\tau_{I} \otimes T I$, as desired.

The right-hand component of (4.33) yields

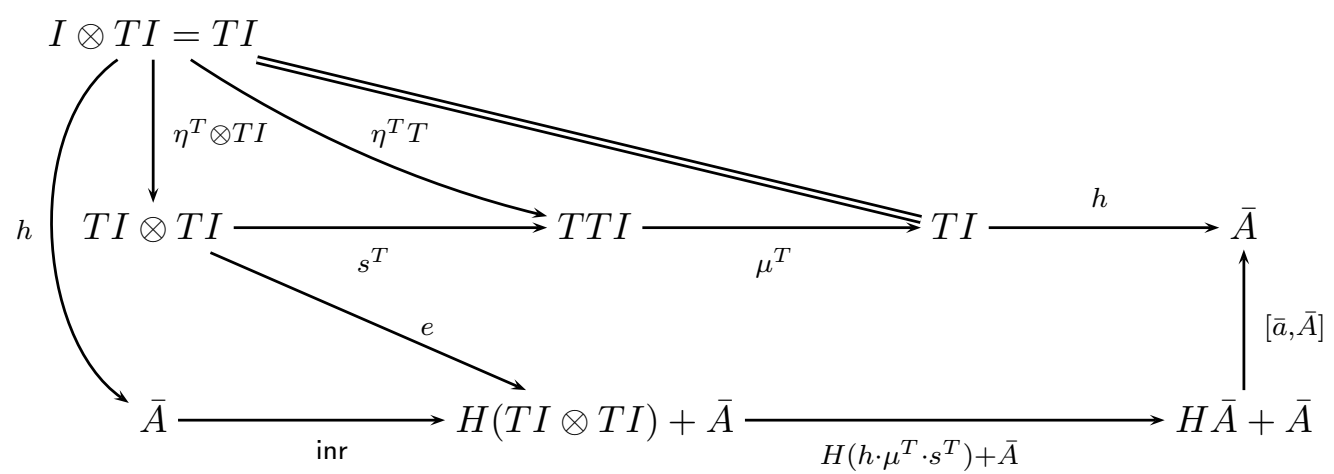

Since all other parts clearly commute we see that the right-hand lower part (4.33) commutes when extended by the coproduct injection $\eta_{I}^{T} \otimes T I$ as desired. For the lower passage of (4.32) we verify that the square

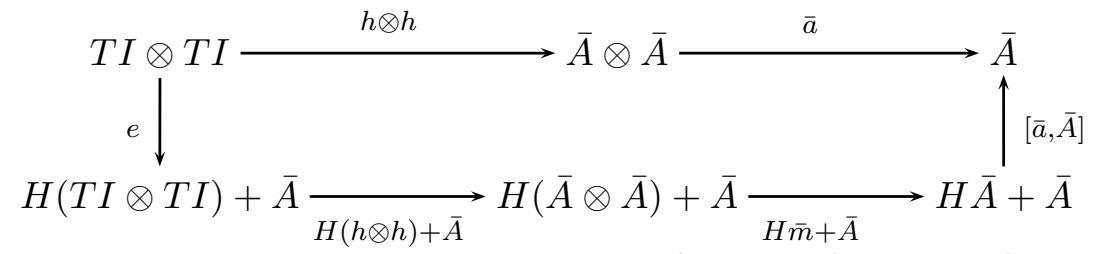

commutes. The left-hand component of $T I \otimes T I=(H T I+I) \otimes T I=(H T I \otimes T I)+T I$ (with coproduct injections $\tau \otimes T I$ and $\eta^{T} \otimes T I$ ) yields

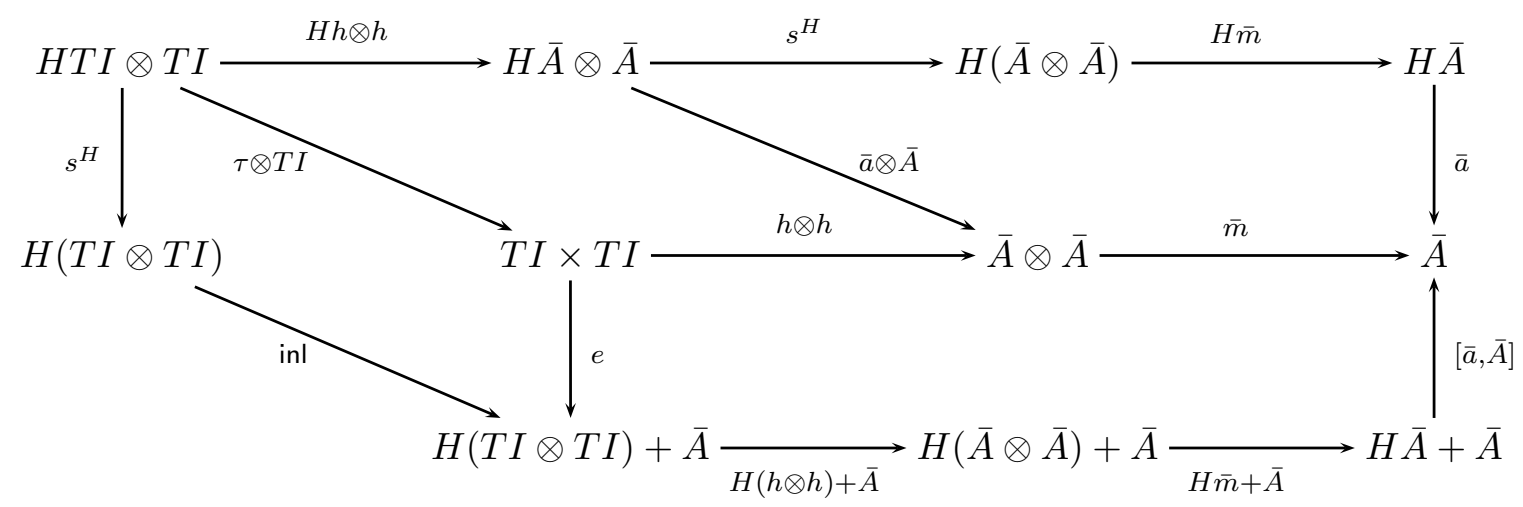

The upper right-hand square is Diagram (3.4) and the middle one commutes since $h$ is an $H$-algebra homomorphism. The left-hand part is clear since $e=s^{H}+h$. The lowest passage in the last diagram is

$$
\bar{a} \cdot H \bar{m} \cdot H(h \otimes h) \cdot s^{H}=\bar{a} \cdot H \bar{m} \cdot s^{H} \cdot(H h \otimes h)
$$

due to the naturality of $s^{H}$. Thus, the outside of the diagram commutes, showing that (4.34) commutes when extended by $\tau_{I} \otimes T I$. 
Finally, the right-hand component of (4.34) is obvious:

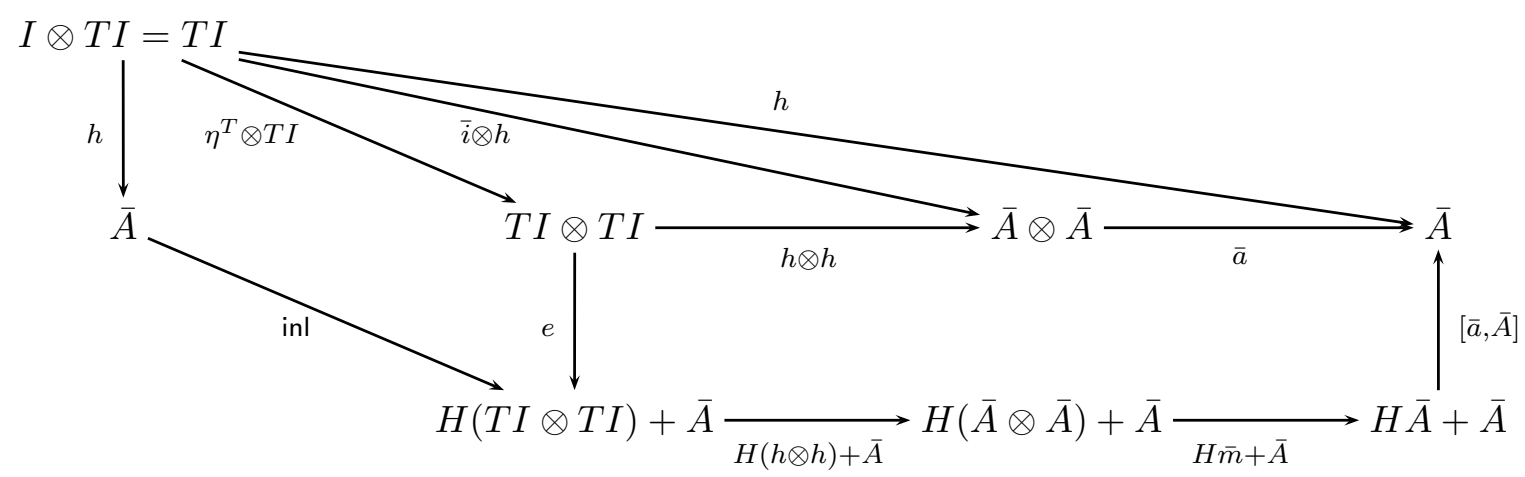

The lower passage is $h$ and so is the upper one:

$$
\bar{a} \cdot(\bar{i} \otimes h)=a \cdot(i \otimes \bar{A}) \cdot(I \otimes h)=I \otimes h .
$$

This shows that (4.34) commutes when extended by $\eta_{I}^{T} \otimes T I$, which completes the proof.

Corollary 4.19. For every signature $\Sigma$ the presheaf $T_{\lambda, \Sigma}$ is the initial completely iterative $H_{\lambda, \Sigma}$-monoid, and the presheaf $R_{\lambda, \Sigma}$ is the initial iterative $H_{\lambda, \Sigma}$-monoid.

Indeed, Set ${ }^{\mathscr{F}}$ is monoidally locally finitely presentable category and $H_{\lambda, \Sigma}$ is finitary and point-strong by Corollary 3.5. Moreover, $\mathbb{R}_{\lambda, \Sigma}(V)=R_{\lambda, \Sigma}$ by Theorem 2.19, and $\mathbb{T}_{\lambda, \Sigma}(V)=T_{\lambda, \Sigma}$ by Theorem 4.18 .

\section{Higher-Order Recursion Schemes}

We can reformulate (and slightly extend) higher-order recursion schemes (1.1) categorically. Throughout this section we put $\mathscr{W}=\operatorname{Set}^{\mathscr{F}}$.

Definition 5.1. A higher-order recursion scheme on a signature $\Sigma$ (of "terminals") is a presheaf morphism

$$
e: X \rightarrow F_{\lambda, \Sigma} \otimes(X+V)
$$

where $X$ is a finitely presentable presheaf.

\section{Remark 5.2.}

(i) The presheaf $F_{\lambda, \Sigma} \otimes(X+V)$ assigns to a context $\Gamma$ the set $F_{\lambda, \Sigma}(X(\Gamma)+\Gamma)$ of finite $\lambda$-terms in contexts $\bar{\Gamma} \subseteq X(\Gamma)+\Gamma$.

(ii) Although $F_{\lambda, \Sigma}$ is the free $H_{\lambda, \Sigma}$-algebra on $V$, see Theorem 2.7, it is not in general true that $F_{\lambda, \Sigma} \otimes Z$ is the free $H_{\lambda, \Sigma}$-algebra on a presheaf $Z$. For example, if $Z=V \times V$, then terms in $\left(F_{\lambda, \Sigma} \otimes Z\right)(\Gamma)$ are precisely the finite $\lambda$ - $\Sigma$-terms whose free variables are substituted by pairs in $\Gamma \times \Gamma$. In contrast, the free $H_{\lambda, \Sigma}$-algebra on $V \times V$ contains in context $\Gamma$ also terms such as $\lambda x .(x, y)$ for $y \in \Gamma$, that is, in variable pairs one member can be bound and one free.

(iii) In the introduction we considered, for a given context

$$
\Gamma_{n t}=\left\{p_{1}, \ldots, p_{n}\right\}
$$

of "nonterminals", a system of equations $p_{i}=f_{i}$, where $f_{i}$ is a $\lambda$ - $\Sigma$-term in some context $\Gamma_{0}=\left\{x_{1}, \ldots, x_{k}\right\}$. Let $X$ be the free presheaf in $n$ generators $p_{1}, \ldots, p_{n}$ of 
context $\Gamma_{0}$ (a coproduct of $n$ copies of $\mathscr{F}\left(\Gamma_{0},-\right)$, see Example 2.2(ii)). Then the system of equations defines the unique morphism

$$
e: X \rightarrow F_{\lambda, \Sigma} \otimes(X+V)
$$

assigning to every $p_{i}$ the right-hand side $f_{i}$ lying in

$$
F_{\lambda, \Sigma}\left(\Gamma_{n t}+\Gamma_{0}\right) \subseteq F_{\lambda, \Sigma}\left(X\left(\Gamma_{0}\right)+\Gamma_{0}\right) .
$$

Here we consider $F_{\lambda, \Sigma}$ as an object of Fin(Set, Set).

(iv) Conversely, every morphism (5.1) yields a system of equations $p_{i}=f_{i}$ as follows: let $\Gamma_{0}$ fulfill (2.6) in Definition 2.14, and define $\Gamma_{n t}=X\left(\Gamma_{0}\right)$. The element $f_{p}=e_{\Gamma_{0}}(p)$ lies, for every nonterminal $p \in \Gamma_{n t}$, in $F_{\lambda, \Sigma}\left(\Gamma_{n t}+\Gamma_{0}\right)$. We obtain a system of equations $p=f_{p}$ describing the given morphism $e$.

(v) We will use the presheaf $R_{\lambda, \Sigma}$ for our uninterpreted solutions of recursion schemes:

A solution of the system of (formal) equations $p_{i}=f_{i}$ are rational $\lambda$ - $\Sigma$-terms $p_{1}^{\dagger}, \ldots, p_{n}^{\dagger}$ making those equations identities in $R_{\lambda, \Sigma}\left(\Gamma_{0}\right)$ when we substitute in $f_{i}$ the $\lambda$ - $\Sigma$-terms $p_{j}^{\dagger}$ for the nonterminals $p_{j}(j=1, \ldots, n)$. This is expressed by the Definition 5.3 below.

(vi) The general case of "equation morphisms" as considered in [2] is (for the endofunctor $\left.H_{\lambda, \Sigma}\right)$ a morphism of type $e: X \rightarrow \mathbb{R}_{\lambda, \Sigma}(X+V)$. We see that every higher-order recursion scheme gives an equation morphism via the inclusion $F_{\lambda, \Sigma} \hookrightarrow R_{\lambda, \Sigma}$ and the strength of the monad $\mathbb{R}_{\lambda, \Sigma}$ (but not necessarily conversely). Our solution theorem below is an application of the general result of [2].

Definition 5.3. A solution of a higher-order recursion scheme $e: X \rightarrow F_{\lambda, \Sigma} \otimes(X+V)$ is a morphism $e^{\dagger}: X \rightarrow R_{\lambda, \Sigma}$ such that the square below, where $j: F_{\lambda, \Sigma} \rightarrow R_{\lambda, \Sigma}$ denotes the embedding, commutes:

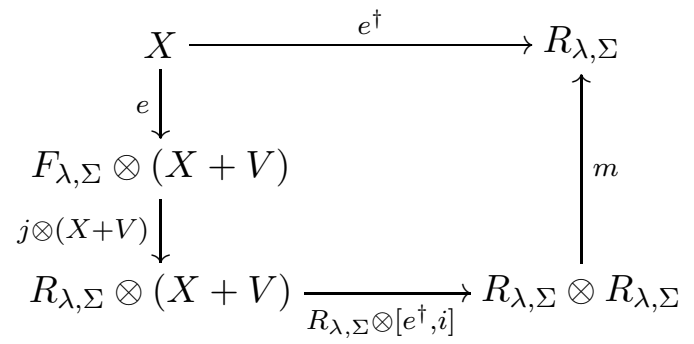

Example 5.4. The equation for the fixed-point combinator (see Example 1.1) with $\Sigma=\emptyset$ defines $e$ whose domain is the terminal presheaf 1 , that is, $e: 1 \rightarrow F_{\lambda} \otimes(1+V)$. The solution $e^{\dagger}: 1 \rightarrow R_{\lambda}$ assigns to the unique element of 1 the tree (1.2).

Remark 5.5. Recursion schemes such as $p_{1}=p_{1}$ make no sense - and they certainly fail to have a unique solution. In general, we want to avoid right-hand sides of the form $p_{i}$. A recursion scheme is called guarded if no right-hand side lies in $\Gamma_{n t}$. (Theorem 5.7 below shows that no other restrictions are needed.) Guardedness can be formalized as follows: since

$$
R_{\lambda, \Sigma}=H_{\lambda, \Sigma}\left(R_{\lambda, \Sigma}\right)+V \quad \text { with injections } \varrho_{V} \text { and } i
$$

by Remark 4.12, we have (see Remark $\underline{3.2}(\mathrm{i})$ )

$$
R_{\lambda, \Sigma} \otimes(X+V) \cong H_{\lambda, \Sigma}\left(R_{\lambda, \Sigma}\right) \otimes(X+V)+X+V
$$


with coproduct injections $\varrho_{V} \otimes \mathrm{id}_{X+V}$ and $i \otimes \mathrm{id}_{X+V}$. Then $e$ is guarded if its extension $(j \otimes(X+V)) \cdot e: X \rightarrow R_{\lambda, \Sigma} \otimes(X+V)$ factorizes through the embedding of the first and third summand of this coproduct:

Definition 5.6. A higher-order recursion scheme $e: X \rightarrow F_{\lambda, \Sigma} \otimes(X+V)$ is called guarded if $(j \otimes(X+V)) \cdot e$ factorizes through

$$
[\varrho \otimes \mathrm{id},(i \otimes \mathrm{id}) \cdot \mathrm{inr}]: H_{\lambda}\left(R_{\lambda, \Sigma}\right) \otimes(X+V)+V \rightarrow R_{\lambda, \Sigma} \otimes(X+V) .
$$

Theorem 5.7. Every guarded higher-order recursion scheme has a unique solution.

Remark. In Definition 5.1 we restricted higher-order recursion schemes to have $F_{\lambda, \Sigma}$ in their codomain. This corresponds well to the classical notion of recursion schemes as explained in Remark 5.2. Moreover, this leads to a simple presentation of the interpreted semantics in Section 6 below. However, Theorem 5.7 remains valid if we replace $F_{\lambda, \Sigma}$ by $R_{\lambda, \Sigma}$ in Definition 5.1 and define solution by $e^{\dagger}=m \cdot R_{\lambda, \Sigma} \otimes\left[e^{\dagger}, i\right] \cdot e$. This extends the notion of a higher-order recursion scheme (1.1) to allow the right-hand sides $f_{i}$ to be rational $\lambda$ - $\Sigma$-terms. We shall prove Theorem 5.7 working with higher-order schemes of the form $e: X \rightarrow R_{\lambda, \Sigma} \otimes(X+V), X$ finitely presentable. We call $e$ guarded if it factorizes through $[\varrho \otimes \mathrm{id},(i \otimes \mathrm{id}) \cdot \mathrm{inr}]$.

Proof. Let us apply the monad $\mathbb{R}_{\lambda, \Sigma}$ and its point-strength $s^{R}$ from Theorem 4.10. We construct for every higher-order recursion scheme $e: X \rightarrow \mathbb{R}_{\lambda, \Sigma}(V) \otimes(X+V)$ a rational equation morphism $\bar{e}: X \rightarrow \mathbb{R}_{\lambda, \Sigma}(X+V)$ in the sense of [2] as follows:

$$
\bar{e} \equiv X \stackrel{e}{\longrightarrow} \mathbb{R}_{\lambda, \Sigma}(V) \otimes(X+V) \stackrel{s_{(V, \text { id })(X+V, \text {,inr })}^{\longrightarrow}}{\longrightarrow} \mathbb{R}_{\lambda, \Sigma}(X+V) .
$$

From the guardedness of $e$ we conclude that $\bar{e}$ is guarded in the sense of [2], that is, $\bar{e}$ factorizes through the summand $H_{\lambda, \Sigma} \mathbb{R}_{\lambda, \Sigma}(X+V)+V$ of $\mathbb{R}_{\lambda, \Sigma}(X+V)$, see Remark 4.12, Indeed, this follows from the following diagram

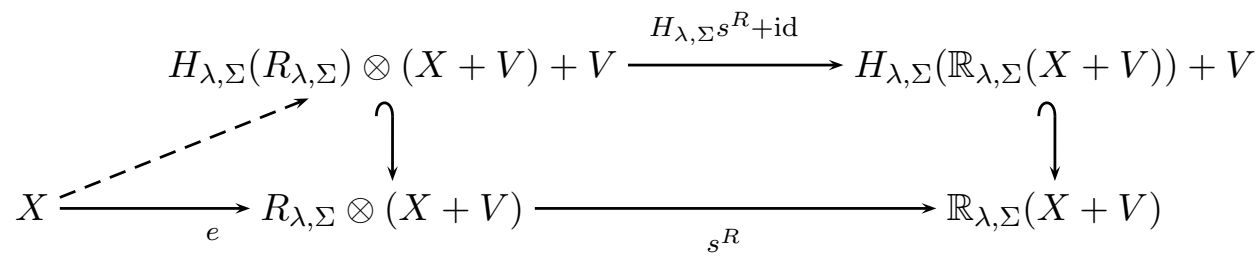

Consequently, by Theorem 4.5 in [2] there exists a unique solution $\bar{e}^{\dagger}$ of $\bar{e}$ with respect to the monad $\mathbb{R}_{\lambda, \Sigma}$ - this means that there exists a unique morphism $\bar{e}^{\dagger}: X \rightarrow \mathbb{R}_{\lambda, \Sigma}(V)$ such that the outside of the diagram below commutes:

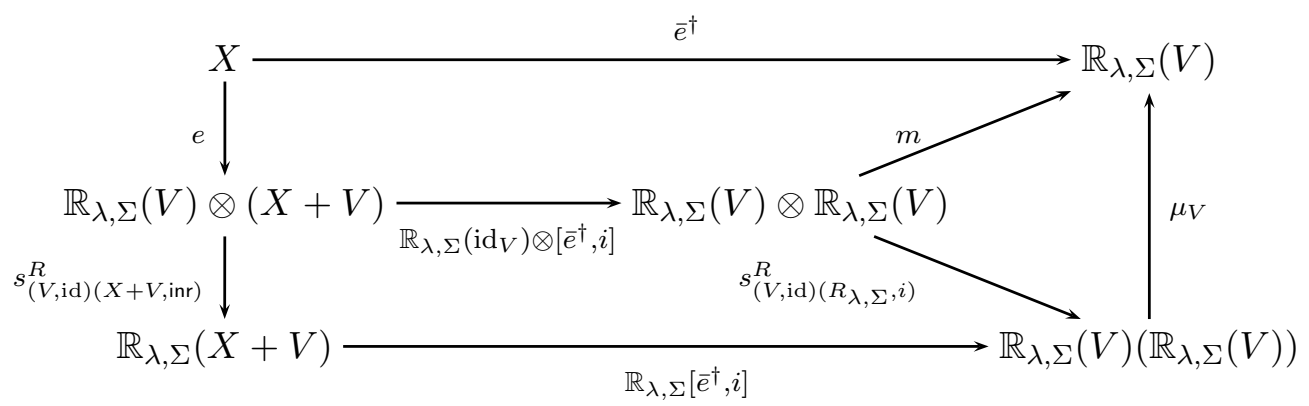


Since the right-hand triangle is Equation (4.20) and the lower square commutes by the naturality of $s^{R}$, we see that $\bar{e}^{\dagger}$ is a solution of $e$ in the sense of Definition 5.3 iff $\bar{e}^{\dagger}$ is a solution of $\bar{e}$ in the sense of [2]. This proves that $e$ has a unique solution.

Remark 5.8. Analogously we could define a solution of a higher-order recursion scheme of the form $e: X \rightarrow T_{\lambda, \Sigma} \otimes(X+V)$ in the initial completely iterative monoid $T_{\lambda, \Sigma}$, see Theorem 3.11. (Here we, moreover, do not need to assume that $X$ is finitely presentable.) And guardedness means here that $e$ factorizes through the summand $H_{\lambda, \Sigma} T_{\lambda, \Sigma} \otimes(X+V)+V$ of $T_{\lambda, \Sigma} \otimes(X+V)$ (cf. Remarks 4.9 and $3.2(\mathrm{i})$ ). Every such guarded scheme has a unique solution in $T_{\lambda, \Sigma}$. The proof is completely analogous to the previous one for $R_{\lambda, \Sigma}$ but using Corollary 3.8 in [1] in lieu of Theorem 4.5 from [2].

Remark 5.9. Notice that the definitions and results in this section generalize to the setting as considered in Section 4 (see Assumption 4.1). Simply replace $H_{\lambda, \Sigma}$ by the finitary functor $H$, the monoid $F_{\lambda, \Sigma}$ by the initial $H$-monoid $F$; this exists and is given by the free $H$-algebra on $V$, see [10]. Further replace the monoid $R_{\lambda, \Sigma}$ by the initial iterative $H$-monoid $R I$ (cf. Theorem 4.17), and $T_{\lambda, \Sigma}$ by the initial completely iterative $H$-monoid (cf. Theorem 4.18).

\section{Interpreted Solutions}

In the present section we prove that every Scott model of $\lambda$-calculus as a CPO, $D$, with fold and unfold operations can be used as a model of higher-order recursion. Following M. Fiore et al [10] we work with the presheaf $\langle D, D\rangle$ which to a context $\Gamma$ assigns the set of all continuous functions from $D^{\Gamma}$ to $D$. We prove that every higher-order recursion scheme has a least solution in $\langle D, D\rangle$.

We denote by CPO the cartesian closed category of posets with directed joins and continuous functions. Thus least elements are not assumed; if they exist we use $\perp$ for them.

Assumption 6.1. We assume that a Scott model $D$ of $\lambda$-calculus is given, i.e., a CPO with $\perp$ and with an embedding-projection pair

$$
\text { fold : } \mathbf{C P O}(D, D) \triangleleft D: \text { unfold . }
$$

Moreover, for the given signature $\Sigma$ of terminals we also assume that continuous operations

$$
\sigma^{D}: D^{n} \rightarrow D \quad \text { for every } n \text {-ary } \sigma \text { in } \Sigma
$$

are given.

Notation 6.2. We define a presheaf $\langle D, D\rangle$ by

$$
\langle D, D\rangle \Gamma=\operatorname{CPO}\left(D^{\Gamma}, D\right) .
$$

\section{Remark 6.3.}

(a) Observe that elements of $\langle D, D\rangle$ can always be interpreted in $D$ : the above function fold: $\langle D, D\rangle 1 \rightarrow D$ yields obvious functions fold $\Gamma:\langle D, D\rangle \Gamma \rightarrow D$ for all contexts $\Gamma$ via induction: define fold $\Gamma+1$ by

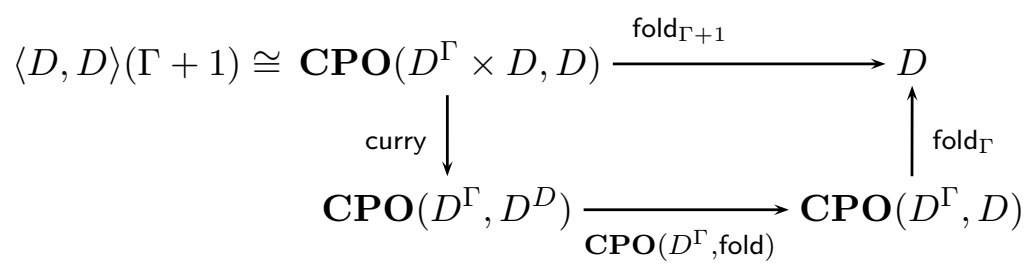


where curry: $\mathbf{C P O}\left(D^{\Gamma} \times D, D\right) \rightarrow \mathbf{C P O}\left(D^{\Gamma}, D^{D}\right)$ is the currification.

(b) The projections $D^{\Gamma} \rightarrow D$ are continuous functions, that is, elements of $\langle D, D\rangle \Gamma$. This defines a natural pointing

$$
\iota: V \rightarrow\langle D, D\rangle
$$

of the presheaf $\langle D, D\rangle$.

Remark 6.4. The presheaf $\langle D, D\rangle$ is an $H_{\lambda, \Sigma}$-monoid. Indeed, application and abstraction are naturally obtained from (6.1), see [10]. The monoid structure

$$
m:\langle D, D\rangle \otimes\langle D, D\rangle \rightarrow\langle D, D\rangle
$$

can be described directly by using the coend formula (3.2) or indirectly:

(a) For the direct description consider the component of $m_{\Gamma}$ corresponding, for an element $f \in \operatorname{Set}\left(\bar{\Gamma}, \mathbf{C P O}\left(D^{\Gamma}, D\right)\right)$, to the injection

$$
\operatorname{in}_{f}: \operatorname{CPO}\left(D^{\bar{\Gamma}}, D\right) \rightarrow \int^{\bar{\Gamma}} \operatorname{Set}\left(\bar{\Gamma}, \operatorname{CPO}\left(D^{\Gamma}, D\right)\right) \bullet \operatorname{CPO}\left(D^{\bar{\Gamma}}, D\right) .
$$

Observe that $f$ yields a continuous function $\tilde{f}: D^{\Gamma} \rightarrow D^{\bar{\Gamma}}$ defined by $\tilde{f}(x)=f(-)(x)$ for all $x \in \Gamma$. We define the component $m_{\Gamma} \cdot \operatorname{in}_{f}$ of $m_{\Gamma}$ by

$$
\langle D, D\rangle \otimes\langle D, D\rangle(\Gamma)=\int^{\bar{\Gamma}} \operatorname{Set}\left(\bar{\Gamma}, \mathbf{C P O}\left(D^{\Gamma}, D\right)\right) \bullet \mathbf{C P O}\left(D^{\bar{\Gamma}}, D\right) \stackrel{m_{\Gamma}}{\longrightarrow} \operatorname{CPO}\left(D^{\Gamma}, D\right)=\langle D, D\rangle \Gamma
$$

(b) There is a much more elegant way of obtaining the monoid structure of $\langle D, D\rangle$. From results of Steve Lack [14] we see that the monoidal category ( $\operatorname{Set}^{\mathscr{F}}, \otimes, V$ ) has the following monoidal action $*$ on $\mathbf{C P O}$ : given $X$ in $\mathbf{S e t}^{\mathscr{F}}$ and $C$ in $\mathbf{C P O}$, we put $X * C=$ $\int^{\Gamma} X(\Gamma) \bullet C^{\Gamma}$. Moreover, extending the above notation to pairs $C, C^{\prime}$ of CPO's and defining $\left\langle C, C^{\prime}\right\rangle \Gamma=\mathbf{C P O}\left(C^{\Gamma}, C^{\prime}\right)$ we obtain a presheaf with a natural isomorphism

$$
\operatorname{Set}^{\mathscr{F}}\left(X,\left\langle C, C^{\prime}\right\rangle\right) \cong \mathbf{C P O}\left(X * C, C^{\prime}\right) \text {. }
$$

As observed by George Janelidze and Max Kelly [13] this yields an enriched category whose hom-objects are $\left\langle C, C^{\prime}\right\rangle$. In particular, $\langle D, D\rangle$ receives a monoid structure. It is tedious but not difficult to prove that this monoid structure is given by (6.2) above and it forms an $H_{\lambda, \Sigma}$-monoid (cf. Definition 3.6).

Notation 6.5. We denote by

$$
\llbracket-\rrbracket: F_{\lambda, \Sigma} \rightarrow\langle D, D\rangle
$$

the unique $H_{\lambda, \Sigma}$-monoid homomorphism (see Theorem 3.9). For every finite term $t$ in context $\Gamma$ we thus obtain its interpretation as a continuous function $\llbracket t \rrbracket_{\Gamma}: D^{\Gamma} \rightarrow D$

Remark 6.6. What is our intuition of an interpreted solution of higher order recursion scheme $e: X \rightarrow F_{\lambda, \Sigma} \otimes(X+V)$ in the presheaf $\langle D, D\rangle$ ? This should be an interpretation of $X$-terms in $\langle D, D\rangle$ via a natural transformation

$$
e^{\dagger}: X \rightarrow\langle D, D\rangle
$$


with the following property: Given an $X$-term $x$ in context $\Gamma$, then $e_{\Gamma}$ assigns to it an element $e_{\Gamma}(x)$ of $\left(F_{\lambda, \Sigma} \otimes(X+V)\right)(\Gamma)$, that is, a finite term $t \in F_{\lambda, \Sigma}(\bar{\Gamma})$ for some $\bar{\Gamma} \subseteq$ $X(\Gamma)+\Gamma$. We request that the solution assigns to $x$ the same value $e_{\Gamma}^{\dagger}(x): D^{\Gamma} \rightarrow D$ that we obtain from the interpretation $\llbracket t \rrbracket_{1}$ of the given term by substituting the $\bar{\Gamma}$-variables using $\left[e^{\dagger}, \iota\right]: X+V \rightarrow\langle D, D\rangle$. This substitution is given by composing $\llbracket-\rrbracket \otimes\left[e^{\dagger}, \iota\right]$ with the monoid structure of $\langle D, D\rangle$. This leads to the following

Definition 6.7. Given a higher-order recursion scheme $e: X \rightarrow F_{\lambda, \Sigma} \otimes(X+V)$ by an interpreted solution is meant a presheaf morphism $e^{\dagger}: X \rightarrow\langle D, D\rangle$ such that the square below commutes:

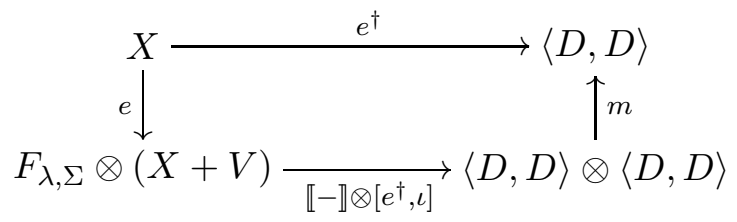

Theorem 6.8. Every higher-order recursion scheme has a least interpreted solution in $\langle D, D\rangle$ in the pointwise ordering of $\operatorname{Set}^{\mathscr{F}}(X,\langle D, D\rangle)$.

Proof. Observe that $\operatorname{Set}^{\mathscr{F}}(X,\langle D, D\rangle)$ is a CPO with $\perp$. Therefore it is sufficient to prove that the endomap of $\operatorname{Set}^{\mathscr{F}}(X,\langle D, D\rangle)$ given by

$$
s \mapsto m \cdot(\llbracket-\rrbracket \otimes[s, \iota]) \cdot e
$$

is continuous, then we can use the Kleene Fixed-Point Theorem. Observe that the function (6.4) is a composite

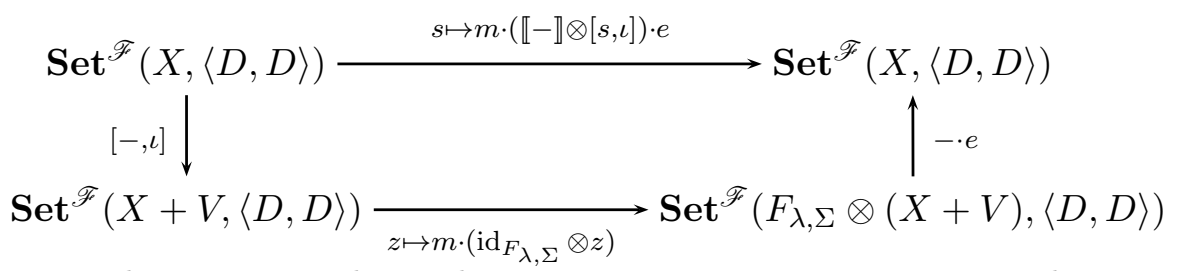

where the vertical arrows are obviously continuous. It remains to prove the continuity of

$$
z \mapsto m \cdot(\mathrm{id} \otimes z) \quad \text { for } z: Z \rightarrow\langle D, D\rangle
$$

where $Z=X+V$ (but this structure of $Z$ plays no role).

We use the coend formula (3.2). It is obvious what the components of

$$
(\operatorname{id} \otimes z)_{\Gamma}: \int^{\bar{\Gamma}} \operatorname{Set}\left((\bar{\Gamma}, Z(\Gamma)) \bullet\langle D, D\rangle \bar{\Gamma} \rightarrow \int^{\bar{\Gamma}} \operatorname{Set}(\bar{\Gamma},\langle D, D\rangle \Gamma) \bullet\langle D, D\rangle \bar{\Gamma}\right.
$$

are: the $\bar{\Gamma}$-component composed with the coproduct injection of $u \in \operatorname{Set}(\bar{\Gamma}, Z(\Gamma))$ yields the coproduct injection of

$$
f=z_{\Gamma} \cdot u \in \operatorname{Set}(\bar{\Gamma},\langle D, D\rangle \Gamma) .
$$

Combined with the component of $m_{\Gamma}$, see (6.2) , this yields the components of $(m \cdot(\mathrm{id} \otimes z))_{\Gamma}$ as follows: for every context $\bar{\Gamma}$ the component composed with the coproduct injection $\mathrm{in}_{u}$ of $u$ is the map

$$
g \mapsto g \cdot \widetilde{z_{\Gamma \cdot u}} \quad \text { for } g \in\langle D, D\rangle \bar{\Gamma} \text {. }
$$


We are ready to prove that the lower horizontal arrow of (6.5) is continuous. Suppose

$$
z=\bigsqcup_{k \in K} z^{k}
$$

is a directed join in the pointwise ordering of $\operatorname{Set}^{\mathscr{F}}(X+V,\langle D, D\rangle)$. In order to prove the equality

$$
m \cdot(\mathrm{id} \otimes z)=\bigsqcup_{k \in K} m \cdot\left(\mathrm{id} \otimes z^{k}\right)
$$

in Set $^{\mathscr{F}}$, we choose an arbitrary context $\Gamma$ and prove that

$$
m_{\Gamma} \cdot\left(\mathrm{id} \otimes z_{\Gamma}\right)=\bigsqcup_{k \in K} m_{\Gamma} \cdot\left(\mathrm{id} \otimes z_{\Gamma}^{k}\right)
$$

holds in Set. For that use the fact that the injection maps, for all contexts $\bar{\Gamma}$ and all $u \in \operatorname{Set}(\bar{\Gamma}, Z(\Gamma))$,

$$
\langle D, D\rangle \bar{\Gamma} \stackrel{\text { in }_{u}}{\longrightarrow} \operatorname{Set}(\bar{\Gamma}, Z(\Gamma)) \bullet\langle D, D\rangle \bar{\Gamma} \stackrel{\text { in }_{\bar{\Gamma}}}{\longrightarrow} \int^{\bar{\Gamma}} \operatorname{Set}(\bar{\Gamma}, Z(\Gamma)) \bullet\langle D, D\rangle \bar{\Gamma}
$$

form a collectively epimorphic cocone. Thus, it is sufficient to prove that for every context $\bar{\Gamma}$ and every $g \in\langle D, D\rangle \bar{\Gamma}$ we have

$$
g \cdot \widetilde{z_{\Gamma} \cdot u}=\bigsqcup_{k \in K} g \cdot \widetilde{z_{\Gamma}^{k} \cdot u}
$$

Since $g$ is continuous from $D^{\bar{\Gamma}}$ to $D$, we just need to verify

$$
\widetilde{z_{\Gamma} \cdot u}=\bigsqcup_{k \in K} \widetilde{z_{\Gamma}^{k} \cdot u}
$$

From the pointwise ordering we clearly get $z_{\Gamma} \cdot u=\bigsqcup_{k \in K} z_{\Gamma}^{k} \cdot u$, thus, we only need to observe the continuity of the map $f \mapsto \tilde{f}$, and this follows from the coordinate-wise ordering of $D^{\Gamma}$.

\section{Conclusions}

We proved that guarded higher-order recursion schemes have a unique uninterpreted solution, i.e., a solution as a rational $\lambda$ - $\Sigma$-term. And they also have the least interpreted solution for interpretations based on Scott's models of $\lambda$-calculus as CPO's with continuous operations for all "terminal" symbols of the recursion scheme.

Following M. Fiore et al [10] we worked in the category Set ${ }^{\mathscr{F}}$ of sets in context, that is, covariant presheaves on the category $\mathscr{F}$ of finite sets and functions. A presheaf is a set dependent on a context (a finite set of variables). For every signature $\Sigma$ of "terminal" operation symbols it was proved in [10] that the presheaf $F_{\lambda, \Sigma}$ of all finite $\lambda$ - $\Sigma$-terms is the initial $H_{\lambda, \Sigma}$-monoid. This means that $F_{\lambda, \Sigma}$ has (i) the $\lambda$-operations (of abstraction and application) together with the operations given by $\Sigma$ rendering an $H_{\lambda, \Sigma^{-}}$-algebra, (ii) the operation expressing simultaneous substitution rendering a monoid in the category of presheaves, and (iii) these two structures are canonically related. And $F_{\lambda, \Sigma}$ is the initial presheaf with such structure. R. Matthes and T. Uutalu [16] showed that the presheaf $T_{\lambda, \Sigma}$ of finite and infinite $\lambda$ - $\Sigma$-terms is also an $H_{\lambda, \Sigma}$-monoid. Here we proved that this is the initial completely 
iterative $H_{\lambda, \Sigma}$-monoid. And its subpresheaf $R_{\lambda, \Sigma}$ of all rational $\lambda$ - $\Sigma$-terms is the initial iterative $H_{\lambda, \Sigma}$-monoid. We used that last presheaf in our uninterpreted semantics of recursion schemes.

Our approach was based on untyped $\lambda$-calculus. The ideas in the typed version are quite analogous. If $S$ is the set of all types, then we form the full subcategory $\mathscr{F}$ of $\mathbf{S e t}^{S}$ of finite $S$-sorted sets and consider presheaves in $\left(\mathbf{S e t}^{S}\right)^{\mathscr{F}}$ - the latter category is equivalent to that of finitary endofunctors of the category $\mathbf{S e t}^{S}$. The definition of $H_{\lambda, \Sigma}$ is then completely analogous to the untyped case, and one can form the presheaves $F_{\lambda, \Sigma}$ (free algebra on $V$ ), $T_{\lambda, \Sigma}$ (free completely iterative algebra) and $R_{\lambda, \Sigma}$ (free iterative algebra). Each of them is a monoid, in fact, an $H_{\lambda, \Sigma}$-monoid in the sense of [10]. Moreover, every guarded higher-order recursion scheme has a unique solution in $R_{\lambda, \Sigma}$. The interpreted semantics can be built up on a CPO-enriched cartesian closed category (as our model of typed $\lambda$-calculus) with additional continuous morphisms for all terminals. The details of the typed version are more involved, and we leave them for future work.

Related results on higher-order substitution can be found e.g. in [16] and [20].

In future work we will, analogously as in [18], investigate the relation of uninterpreted and interpreted solutions.

\section{REFERENCES}

[1] Aczel, P., Adámek, J., Milius, S., Velebil, J.: Infinite trees and completely iterative theories: a coalgebraic view. Theoret. Comput. Sci. 300 (2003), 1-45.

[2] Adámek, J., Milius, S., Velebil, J.: Iterative algebras at work. Math. Structures Comput. Sci. 16 (2006), 1085-1131.

[3] Adámek, J., Milius, S., Velebil, J.: Elgot Algebras. Log. Methods Comput. Sci. 2(5:4), 2006,32 pp.

[4] Adámek, J., Milius, S., Velebil, J.: Semantics of higher-order recursion schemes. Proc. Algebra and Coalgebra in Computer Science (CALCO 2009), Lect. Notes Comput. Sci. 5728, Springer 2009, 49-63.

[5] Adámek, J., Porst, H. E.: On tree coalgebras and coalgebra presentations. Theoret. Comput. Sci. 311 (2004), 257-283.

[6] Adámek, J., Trnková, V.: Automata and algebras in a category. Kluwer Academic Publishers, Dordrecht, 1990.

[7] Aehlig, K.: A finite semantics of simply-typed lambda terms for infinite runs of automata. Proc. Computer Science Logic 2006, Lect. Notes Comput. Sci. 4207, Springer 2006, 104-118.

[8] Damm, W.: Higher-order program schemes and their languages. Lect. Notes Comput. Sci. 48, Springer 1979, 51-72.

[9] Fiore M.: Second order dependently sorted abstract syntax. Proc. Logic in Computer Science 2008, IEEE Press 2008, 57-68.

[10] Fiore, M., Plotkin, G., Turi, D.: Abstract syntax and variable binding. Proc. Logic in Computer Science 1999, IEEE Press 1999, 193-202.

[11] Garland, S. J., Luckham, D. C.: Program schemes, recursion schemes and formal languages. J. Comput. Syst. Sci. 7 (1973), 119-160.

[12] Guessarian, I.: Algebraic semantics. Lect. Notes Comput. Sci. 99, Springer, 1981.

[13] Janelidze, G., Kelly, G. M.: A note on actions of a monoidal category. Theory Appl. Categ. 9 (2001), 61-91

[14] Lack, S.: On the monadicity of finitary monads. J. Pure Appl. Algebra 140 (1999), 65-73.

[15] Makkai, M., Paré R.: Accessible Categories: The Foundations of Categorical Model Theory. Contemporary Mathematics Vol. 104, American Mathematical Society, 1989.

[16] Matthes, R., Uustalu, T.: Substitution in non-wellfounded syntax with variable binding. Theoret. Comput. Sci. 327 (2004), 155-174.

[17] Milius, S.: Completely iterative algebras and completely iterative monads. Inform. and Comput. 196 (2005), 1-41. 
[18] Milius, S., Moss, L.: The category theoretic solution of recursive program schemes. Theoret. Comput. Sci. 366 (2006), 3-59, corrigendum in 403 (2008), 409-415.

[19] Miranda, G.: Structures generated by higher-order grammars and the safety constraint. Ph.D. Thesis, Merton College, Oxford, 2006.

[20] Power, J.: A unified category theoretical approach to variable binding. Proc. MERLIN 2003. 